\title{
Variability Analysis via Parametrized Model Order Reduction
}

\author{
by \\ Ye Tao

\begin{abstract}
A thesis submitted to the
Faculty of Graduate and Postdoctoral Affairs

in partial fulfillment of the requirements for the degree of
\end{abstract}

Master of Applied Science in Electrical Engineering

Ottawa-Carleton Institute for Electrical and Computer Engineering

Department of Electrical Engineering

Carleton University

Ottawa, Ontario

August, 2016

(C)Copyright

Ye Tao, 2016 
The undersigned hereby recommends to the

Faculty of Graduate and Postdoctoral Affairs acceptance of the thesis

\title{
Variability Analysis via Parametrized Model Order Reduction
}

\author{
submitted by Ye Tao \\ in partial fulfillment of the requirements for the degree of
}

Master of Applied Science in Electrical Engineering

Professor Michel Nakhla, Thesis Supervisor

Professor Ram Achar, Thesis Co-supervisor

Dr. Behzad Nouri, Thesis Co-supervisor

Professor Niall Tait, Chair, Department of Electrical Engineering

Ottawa-Carleton Institute for Electrical and Computer Engineering

Department of Electrical Engineering

Carleton University

August, 2016 


\section{Abstract}

With the continuously decreasing feature sizes, variability analysis of nano-scale highspeed and low-power circuits are becoming increasingly important. In this thesis, two efficient algorithms are presented for statistical analysis of high-speed circuits with multiple stochastic parameters in both frequency and time domains.

In the frequency domain, using the proposed algorithm, a set of local reduced-order parameterized circuits are derived via an implicit multi-moment matching projection technique. The local models preserve the stochastic parameters as symbolic quantities. As a result, stochastic response of the circuit can be obtained by simulating the local reduced models instead of the original large system leading to significant reduction in the computational cost compared to traditional Monte-Carlo techniques.

In the time domain, the proposed method combines the merits of the parametrized model order based techniques and Numerical Inversion of Laplace Transform (NILT). The response of the reduced-model at any given time point is expressed as a linear combination of the frequency domain response at relatively small number of predetermined complex frequency points. This eliminates the necessity for explicit representation of the dynamic model in the form of a set of differential equations. As a result, the moment vectors associated with frequency are excluded while forming the moments subspace, leading to much smaller reduced-models. In addition, evaluation of the time-domain response of the reduced-order models using NILT is more efficient 
and highly parallelizable compared to time-stepping numerical integration techniques. Numerical examples are presented to demonstrate the efficiency and accuracy of the proposed method. 


\section{Acknowledgments}

My deepest gratitude goes first and foremost to Professor Michel Nakhla, my supervisor, for his constant encouragement, unconditioned support and invaluable advice. With his guidance, the research has been an enjoyable adventure. Never could I become a great person with infinite knowledge as he is.

I would like to express my heartfelt gratitude to Prof. Ram Achar and Dr. Behzad Nouri, who not only shared their knowledge with me, but also had great patience with my writings, without whom the thesis could not have been possible. I also thank Prof. Achar for the great lecture, in whose class I met my future wife Phoebe.

Also, my thanks would go to the CAD team, Kai, Henry and especially Mina, who guided my fourth year project and my first project in graduate study. All the associated interesting discussions inspired me into this work. They are truly great friends. The friendship will last much beyond this endeavor.

I would like to give special thanks to my fiancée, Phoebe, for all her support and understanding. A home is so desperately needed for a voyager alone in a foreign country. A home with her is a home.

I would also like to express my deep love to my parents for their unconditioned love and care. Without their continuous encouragement and support, I could never reached this far. To them, I dedicate this thesis. 


\section{Contents}

Abstract $\quad$ iii

Acknowledgments $\quad$ v

Table of Contents vi vi

List of Tables $\quad$ ix

List of Figures $\quad$ X

List of Acronyms xii

List of Symbols $\quad$ xiv

1 Introduction 1

1.1 Motivation and Background . . . . . . . . . . . . 1

1.2 Objectives and Contributions . . . . . . . . . . . . . 3

1.3 Organization of the Thesis . . . . . . . . . . . . . . 4

2 Preliminaries $\quad 5$

2.1 Formulation of Circuit Equations . . . . . . . . . . . . 5

2.1.1 Linear-Circuit Equations . . . . . . . . . . . . 6

2.1.2 Time-Domain Analysis using Time-Stepping methods . . . . . 7 
2.2 Model-Order Reduction for Linear Circuits . . . . . . . . . . . . . . . 9

2.2.1 Asymptotic Waveform Evaluation (AWE) . . . . . . . . . 9

2.2.2 Arnoldi Algorithm . . . . . . . . . . . . . . . . . . . 11

2.2 .3 PRIMA . . . . . . . . . . . . . . . . . . . . . . . 12

2.3 Discussion . . . . . . . . . . . . . . . . . . 13

3 Review of Parametric Model Order Reduction $\quad 14$

3.1 Introduction . . . . . . . . . . . . . . . . . . 14

3.2 Multi-Dimensional Modeling . . . . . . . . . . . . . . . . . . . . . 14

3.3 Explicit Parametric Moment-Matching Model-Order Reduction . . . . 17

3.4 Implicit Parametric Model-Order Reduction . . . . . . . . . . . . . . 19

3.5 Applications using Parametric Model-Order Reduction . . . . . . . . 21

3.6 Difficulties with Conventional PMOR . . . . . . . . . . . . . 23

4 Proposed Variability Analysis using PMOR in Frequency Domain 25

4.1 Concept Associated with Proposed PMOR . . . . . . . . . . . 25

4.2 Case of Single Random Parameter . . . . . . . . . . . . . . . . . 31

4.3 Case of Multiple Parameters . . . . . . . . . . . . . . . 36

4.3 .1 Discussion . . . . . . . . . . . . . . . . . . 41

4.4 Proposed Parametric Model-Order Reduction Using Implicit Moment Matching . . . . . . . . . . . . . . . . . 42

4.5 Numerical Examples . . . . . . . . . . . . . . . . . . 45

4.5.1 Example I . . . . . . . . . . . . . . . 45

4.5.2 Example II . . . . . . . . . . . . . . . . . . . . . . . 47

5 Proposed Time Domain Variability Analysis Using PMOR and $\begin{array}{ll}\text { NILT } & 50\end{array}$ 
5.1 Concept of the Proposed Method . . . . . . . . . . . . . 50

5.2 Numerical Inversion of Laplace Transform _ . . . . . . . . . . 52

5.3 Time-domain Statistical Analysis using NILT . . . . . . . . . 55

5.4 Orthogonal Matrix Calculation _. . . . . . . . . . 57

5.5 Time-domain Variability Analysis Using PMOR and NILT . . . . . 60

5.6 Numerical Examples . . . . . . . . . . . . . . . . . . . . . . 63

$5.6 .1 \quad$ Example I . . . . . . . . . . . . . . . . . . . . . . 63

5.6 .2 Example II . . . . . . . . . . . . . . . . 67

5.6 .3 Example III . . . . . . . . . . . . . . . . 70

6 Conclusions and Future Work $\quad 76$

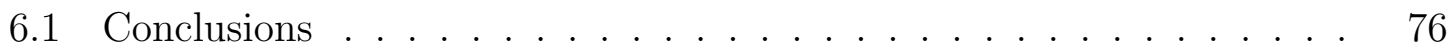

6.2 Future Work . . . . . . . . . . . . . . . . . . . . . 78

$\begin{array}{ll}\text { List of References } & 79\end{array}$

$\begin{array}{ll}\text { Appendix A Arnoldi Algorithm } & 83\end{array}$

$\begin{array}{ll}\text { Appendix B Block Arnoldi Algorithm } & 84\end{array}$

Appendix C Orthogonal Matrix Calculation for Parametric System 85

$\begin{array}{ll}\text { Appendix D Stability of NILT } & 86\end{array}$

$\begin{array}{lll}\text { Appendix E } & \text { Error Estimation of NILT } & 88\end{array}$ 


\section{List of Tables}

3.1 Problem Sizes and CPU computation time for Conventional PMOR . 22

4.1 CPU time and Size Comparison (Example 1) . . . . . . . . . . 47

4.2 CPU time and Size Comparison (Example 2) . . . . . . . . . . 48

5.1 CPU time and Size Comparison (Example 1) . . . . . . . . . 64

5.2 CPU time and Size Comparison (Example 2) . . . . . . . . 70

5.3 CPU time and Size Comparison (Example 3) . . . . . . . . . 71 


\section{List of Figures}

3.1 Four coupled interconnects circuit . . . . . . . . . . . . . . . 21

3.2 Statistical analysis for the magnitude of the far-end voltage of active line(Example in Section 3.5) . . . . . . . . . . . . . . . . 22

4.1 Response of active line of an interconnect circuit w.r.t. frequency and width of lines . . . . . . . . . . . . . . . . . 27

4.2 Response of victim line of an interconnect circuit w.r.t. frequency and width of lines . . . . . . . . . . . . . . . . . . . 28

4.3 Response of active line of an interconnect circuit at $3 \mathrm{GHz}$ w.r.t. width of lines and separations between lines. . . . . . . . . . . . . . 29

4.4 Response of victim line of an interconnect circuit at $3 \mathrm{GHz}$ w.r.t. width of lines and separations between lines. . . . . . . . . . . . . 30

4.5 Four coupled interconnects circuit . . . . . . . . . . . . . . 45

4.6 Statistical analysis for the voltage waveform at the far-end of active line \#1 (Example 1). . . . . . . . . . . . . . . . . 46

4.7 Circuit for Example 2 (Example 2$) \ldots \ldots \ldots \ldots$

4.8 Statistical analysis for the voltage waveform at the far-end of active line \#1 (Example 2) . . . . . . . . . . . . . . . . . . . . . . . . . . . 49

5.1 An illustration of NILT that the time response at one time point is a summation of frequency responses at several complex frequencies. . 
5.2 (a) Four coupled interconnects circuit, (b) Excitation waveform at input (Example 1). . . . . . . . . . . . . . . . . . 64

5.3 Statistical analysis for the voltage waveform at the far-end of active

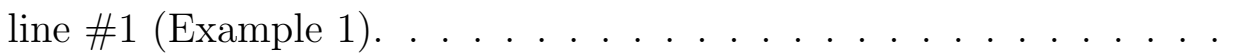

5.4 Statistical analysis for the voltage waveform at the far-end of victim line \#2 (Example 1) . . . . . . . . . . . . . . . 66

5.5 Four coupled interconnects circuit (Example 2) $\ldots \ldots \ldots$. . . 67

5.6 Statistical analysis for the voltage waveform at the far-end of active line \#1 (Example 2). . . . . . . . . . . . . . . . . 68

5.7 Statistical analysis for the voltage waveform at the far-end of victim line \#2 (Example 2) . . . . . . . . . . . . . . . . . . . . . . 69

5.8 Eight coupled interconnects circuit (Example 3) . . . . . . . . . . 71

5.9 Statistical analysis for the voltage waveform at the far-end of active line \#1 (Example 3). . . . . . . . . . . . . . . . . . . . . . . . 72

5.10 Statistical analysis for the voltage waveform at the far-end of victim line \#2 (Example 3$). \ldots \ldots$. . . . . . . . . . . . . . . 73

5.11 Statistical analysis for the voltage waveform at the far-end of active line \#5 (Example 3). . . . . . . . . . . . . . . . . . . . 74

5.12 Statistical analysis for the voltage waveform at the far-end of victim line \#6 (Example 3$) \ldots \ldots \ldots$. . . . . . . . . . . . . . 75 


\section{List of Acronyms}

\section{Acronyms Definition}

\begin{tabular}{ll}
\hline \hline MC & Monte Carlo Simulation \\
EM & Electromagnetic \\
MOR & Model-Order Reduction \\
ROM & Reduced-Order Model \\
CAD & Computer Aided Design \\
MNA & Modified Nodal Analysis \\
KCL & Kirchoff's Current Law \\
IFFT & inverse Fast-Fourier Transform \\
DEs & Differential Equations \\
LMS & Linear Multi-Step Methods \\
VLSI & Very-Large-Scale Integration \\
AWE & Asymptotic Waveform Evaluation
\end{tabular}


SISO Single Input Single Output

MIMO Multiple Input Multiple Output

PRIMA Passive Reduced-Order Interconnect Macro-Modeling Algorithm

PMOR Parametrized Model-Order Reduction

NILT Numerical Inversion of Laplace Transform 


\section{List of Symbols}

\section{Symbols Definition}

G matrix describing the memoryless elements in the circuit

C matrix describing the memory elements in the circuit

$\mathbf{X}(s) \quad \begin{aligned} & \text { vector of the voltages of circuit nodes, currents in the inductors or independent voltage } \\ & \text { sources }\end{aligned}$ sources

L a selector matrix for the output port of the circuit

B matrix describing the input elements in the circuit

$H(s) \quad$ transfer function of a linear circuit

$\mathcal{Q} \quad$ orthogonal basis

H upper-Hessenberg matrix

$\xi \quad$ set of multi-random variable

$\mathbf{M}_{\xi_{i}} \quad$ moments with respect to parameter $\xi_{i}$

f $\quad$ frequency

I identity matrix 
$\Re$ or $\Re e \quad$ real part of a complex number

$\hat{\mathbf{G}}, \hat{\mathbf{C}}, \hat{\mathbf{B}}, \hat{\mathbf{L}} \quad$ reduced-order model of $\mathbf{G}, \mathbf{C}, \mathbf{B}, \mathbf{L}$ 


\section{Chapter 1}

\section{Introduction}

\subsection{Motivation and Background}

According to Moore's Law, the number of transistors in a dense integrated circuit doubles approximately in every two years. Nowadays, the device dimensions are in nano-scale region while the signals are in the $\mathrm{GHz}$ range. Due to the increasing demand for highly operating frequencies, the effects of the interconnects, such as delay, distortion, crosstalk and reflections, etc, become the dominant factors limiting the performance. In order to capture these effects, designers desire accurate circuit simulations at a low CPU computational cost.

One of the main challenges facing designers of nano-scaled high-speed integrated circuits and interconnects is predicting the effect of the variability of geometrical and physical parameters on the circuit performance. Monte Carlo (MC) simulation has been traditionally used in commercial circuit and electromagnetic (EM) simulators for predicting the statistical distribution of circuit and system performance metrics. However, the high computational cost associated with the MC simulation is a significant limiting factor especially for large circuits. This fact has prompted wide interest in exploring alternative approaches. 
Model-order reduction (MOR) has demonstrated great efficiency in simulating large-scale systems. The reduction algorithm is based on projecting the original large set of equations on a lower dimension space using orthogonal congruence transformation. The most commonly used projection approach for non-parametric systems is based on moment matching which preserves certain number of the leading terms of Taylor series expansion of the response with respect to frequency. To achieve this, a projection matrix is formed using the orthogonal basis of the Krylov subspace spanned by the moment vectors with respect to frequency [1].

In addition, the MOR concept has been extended to include multi-moment matching with respect to frequency as well as other design parameters, such as geometrical layout or substrate characteristics [2-4]. In this case, a global basis may be computed for the subspace spanned by the moment vectors associated with frequency as well as the stochastic parameters. One obvious drawback of the global basis approach is that the size of the reduced-order model in general, is relatively large leading to small speed-up over solving the original full system. Also, in the case of variability analysis in the time-domain, the common goal of traditional PMOR techniques [2-4] is to obtain a dynamic reduced macromodel in the form of a set of differential equations. This necessitates the inclusion of the moment vectors associated with the frequency which causes substantial increase in the size of the reduced order model (ROM). 


\subsection{Objectives and Contributions}

The goal of this thesis is to present an efficient algorithm for statistical analysis of large circuits with multiple stochastic parameters via parametrized model order reduction. A new PMOR method has been developed for variability analysis in both frequency domain and time domain. The proposed method offers the following important advantages.

- In the proposed method, moment vectors associated with frequency are excluded while forming the moments subspace. This leads to much smaller reduced-ordermodels, since the variation of the circuit response with respect to stochastic parameters, in general is much smoother than the variation with respect to frequency. Also, the new method provides for implicit computation of moments with respect to stochastic parameters $[5,6]$.

- Unlike the previous PMOR methods, the proposed algorithm does not require an explicit presentation of the dynamic model in the form of a set of differential equations. Instead, by appropriately adapting the NILT algorithm, the circuit response at any given time point is expressed as a linear combination of the frequency-domain response at relatively small number of pre-determined complex frequency points $[5,6]$.

The proposed algorithms are highly parallelizable compared to time-stepping numerical integration techniques, leading to significant speed-up. 


\subsection{Organization of the Thesis}

In the rest of this thesis, Chapter 2 briefly reviews the background of circuit equations and model-order reductions (MOR) algorithms. Chapter 3 describes the conventional parametrized model order reduction (PMOR) with an numerical example and shows the difficulties of applying the conventional PMOR to variability analysis. Chapter 4 presents the proposed efficient PMOR method for variability analysis in frequency domain with two numerical examples validating the accuracy and speed-up. Chapter 5 provides a new method combining Numerical Inversion of Laplace Transform with the

proposed PMOR process. Conclusions and future work are presented in chapter 6 . Several related algorithms and mathematical proofs are provided in the Appendices. 


\section{Chapter 2}

\section{Preliminaries}

This chapter presents a brief background of circuit simulation as relevant to this thesis. The formulation of circuit equations is provided in Section 2.1. The concept of model order reduction (MOR) is described in Section 2.2.

\subsection{Formulation of Circuit Equations}

One of the well known formulation in computer-aided design (CAD) is Modified Nodal Analysis (MNA). The idea of MNA formulation is to evaluate the voltage at each nodes and current in some specific branches of the circuit by forming the equations in matrices following Kirchoff's Current Law (KCL). Components of the circuit, such as resistors, capacitors, inductors, op-amp ect., can be translated into stamps. Each stamp is representing the effect of components by manipulating the appropriate entries in the matrices. This process is called MNA formulation.

The MNA formulation has been widely used in computer-aided design(CAD). Any SPICE like simulators will translate the netlist descriptor which lists all the stamps of circuit into MNA formulation, then the dynamic behavior of the circuit over time can be simulated by solving the MNA equation. 


\subsubsection{Linear-Circuit Equations}

A linear circuit is that all the components in the circuit are linear to frequency and Kirchoff's laws also hold. If the outputs of a circuit is a function $\mathcal{L}$ given any input $u_{i}$, a linear circuit will satisfy with the following properties:

- $\mathcal{L}\left\{u_{1}+u_{2}\right\}=\mathcal{L}\left\{u_{1}\right\}+\mathcal{L}\left\{u_{2}\right\}$ (Additivity)

- $\mathcal{L}\left\{K u_{i}\right\}=K \mathcal{L}\left\{u_{i}\right\}$ (Homogeneity)

where $\mathrm{K}$ is an arbitrary real number.

The MNA formulation for linear circuit in time domain is defined as:

$$
\begin{aligned}
\mathbf{G} x(t)+\mathbf{C} \frac{d x}{d t} & =\mathbf{B} u(t) \\
v(t) & =\mathbf{L}^{t} x(t)
\end{aligned}
$$

where $\mathbf{C}$ and $\mathbf{G} \in \mathbb{R}^{N \times N}$ are susceptance and conductance matrices, respectively, $x(t) \in \mathbb{R}^{N}$ denotes the vector of MNA variables (the nodal voltages and some branch currents) of the circuit. Also, $\mathbf{B} \in \mathbb{R}^{N \times p}$ and $\mathbf{L} \in \mathbb{R}^{N \times q}$ are the input and output matrices, associated with p inputs and q outputs, respectively.

Equation (2.1) in Laplace domain can be easily obtained by a Laplace transformation. Given an initial state $x(0)$, equation (2.1) becomes

$$
\begin{aligned}
(\mathbf{G}+s \mathbf{C}) X(s)+\mathbf{C} x(0) & =\mathbf{B} U(s) \\
V(s) & =\mathbf{L}^{t} X(s),
\end{aligned}
$$

where $\mathrm{s}$ is the Laplace operator.

Assuming zero initial conditions, the MNA formulation in Laplace domain can be 
obtained as:

$$
\begin{aligned}
(\mathbf{G}+s \mathbf{C}) X(s) & =\mathbf{B} U(s) \\
V(s) & =\mathbf{L}^{t} X(s)
\end{aligned}
$$

It is to be noticed that (2.1) is a first order differential equation with state variable $x(t)$. The trajectory of $x(t)$, or in other words the response of circuit, can be obtained in either time domain or frequency domain. Integration methods such as linear-multistep methods in [7] or high-order integration methods in [8-11] can be used to obtain the response in time domain. The common goal of the integration methods in time domain is to solve (2.1) iteratively given an initial condition $x(t=0)$. This process is called transient analysis in circuit simulation.

Another category of time-domain analysis is based on analyzing the circuit in frequency domain or in Laplace domain, wherein, the frequency response is obtained by solving (2.3) at each frequency point. Subsequently, the time-domain response can be calculated using inverse Fast-Fourier Transform (IFFT).

\subsubsection{Time-Domain Analysis using Time-Stepping methods}

TIME-DOMAIN simulators represent an important category of design automation tools and are essential for analysis and validation of electronic circuits. Designers of electronic circuits typically use transient analysis tools for doing verification purposes and expect it to provide superior accuracy while requiring shorter execution times and limited computational resources. Typically, these requirements are often in conflict with each other for most applications and present far more serious hurdles while performing circuit-level transient analysis. The primary cause of such a conflict arises while solving underlying differential equations (DEs). 
One of the main category of time domain simulations is to solve the differential equations using numerical integrating techniques. Linear Multi-Step (LMS) Methods, such as backward-euler and trapezoidal rule, are widely used in current commercial packages in traditional DAE solvers [7]. However LMS methods suffer from the inherent conflict between "order" and "stability".

The order of the method reflects the computational effort needed to achieve a better accuracy. However, high-accuracy integration using a low-order method necessitates a significant reduction in the step size, causing solutions to be performed at more number of time points. Another issue is the evaluation of nonlinear devices (and its derivatives) at each time step, where in the modern devices require more CPU time to evaluate. Typically, the cost associated with such evaluation dominates the computational efforts spent per time step. Both these issues often cause transient analysis times to run excessively longer. Hence, high-order methods which naturally lead to a reduction in the number of time steps would therefore be crucial to efficient simulations. However, as has been established by the second Dahlquist barrier [12], the highest achievable order in this class of methods does not exceed order 2, beyond which the stability of numerical integration cant be guaranteed. In order to address this difficulty, recently a new class of higher order integration method based on Obreshkovs formula was developed [8-11] guaranteeing both the A-stablity as well as L-stablity. 


\subsection{Model-Order Reduction for Linear Circuits}

As the technology of integrated circuit and VLSI circuit grows rapidly nowadays, one of the major challenges faced by the circuit simulators is how to minimize the simulation cost for large-scale circuits. Model-order Reduction algorithms provide elegant solutions to this challenges [13-17]. The idea of model-order reduction is that, given a large-scale circuit, a small-scale system can be found such that the behavior of the small system can reasonably accurately approximate the large system. There are a number of methods for linear model reduction and two main categories are reviewed in the following sections.

\subsubsection{Asymptotic Waveform Evaluation (AWE)}

The AWE technique for linear circuit was first reported in [18]. By a transformation, the output of $(2.3) V(s)$ is related to the input $U(s)$ as:

$$
V(s)=\mathbf{L}^{t}(\mathbf{G}+s \mathbf{C})^{-1} \mathbf{B} U(s),
$$

which is in the form of:

$$
V(s)=\mathbf{H}(s) U(s) .
$$

Therefore given a zero input, the transfer function of a linear circuit is defined as:

$$
H(s)=L^{t}(\mathbf{G}+s \mathbf{C})^{-1} \mathbf{B} .
$$

A further transformation can be applied to the transfer function as:

$$
H(s)=L^{t}(\mathbf{I}-s \mathbf{A})^{-1} \mathbf{R}
$$


by defining $\mathbf{R}=\mathbf{G}^{-1} \mathbf{B}$ and $\mathbf{A}=-\mathbf{G}^{-1} \mathbf{C}$.

The transfer function $H(s)$ can be expanded using Taylor series at any complex frequency point $s_{0}$ as:

$$
\begin{aligned}
H(s) & =L^{t}\left(\mathbf{R}+\left(s-s_{0}\right) \mathbf{A} \mathbf{R}+\left(s-s_{0}\right)^{2} \mathbf{A}^{2} \mathbf{R}+\ldots+\left(s-s_{0}\right)^{n} \mathbf{A}^{n} \mathbf{R}+\ldots\right) \\
& =\sum_{n=0}^{\infty}\left(s-s_{0}\right)^{n} L^{t} \mathbf{A}^{n} \mathbf{R}
\end{aligned}
$$

The coefficients $L^{t} \mathbf{A}^{n} \mathbf{R}$ are called the $n^{\text {th }}$ order moments of the transfer function.

Asymptotic waveform evaluation (AWE) algorithm [19] was based on Padé approximation of the transfer function of (2.8). The reduced-order rational function matches the leading moments up to order of $m$ by explicitly calculating the leading $2 m$ moments of (2.8). This method was first widely used in interconnect modeling. However, the major drawback of this method is that, generating the moments explicitly becomes numerical unstable when the order of moments becomes larger. In order to generate the $n^{\text {th }}$ order moments, the calculation of $\mathbf{A}^{n}$ converges quickly to the eigenvector associated with the eigenvalue having the largest magnitude. In the end, the moments vectors become all linearly dependent to this eigenvector [18]. 


\subsubsection{Arnoldi Algorithm}

To address the difficulties associated with the explicit moment matching, reduction algorithms based on Krylov-based projection methods were proposed. For projection methods, the original system in (2.3) is projected into a reduced-order model (ROM) in the following form:

$$
(\tilde{\mathbf{G}}+s \tilde{\mathbf{C}}) \tilde{\mathbf{X}}(s)=\tilde{\mathbf{B}} U(s)
$$

where

$$
\begin{aligned}
\tilde{\mathbf{G}} & =\mathcal{Q}^{t} \mathbf{G} \mathcal{Q}, \\
\tilde{\mathbf{C}} & =\mathcal{Q}^{t} \mathbf{C} \mathcal{Q}, \\
\tilde{\mathbf{B}} & =\mathcal{Q}^{t} \mathbf{B} .
\end{aligned}
$$

Then the output response of the original system in (2.3) can be obtained from the $\mathrm{ROM}$ as

$$
\mathbf{X}(s)=\mathcal{Q} \tilde{\mathbf{X}}(s)
$$

In order to generate the orthogonal operator $\mathcal{Q}$, methods in $[15,20]$ are introduced. Take a closer look at the moments vectors of the transfer function (2.8), the subspace of the moments vectors is in the form of:

$$
\operatorname{colsp}\{\mathcal{Q}\}=\left[\mathbf{R}, \mathbf{A R}, \mathbf{A}^{2} \mathbf{R}, \ldots, \mathbf{A}^{n} \mathbf{R}, \ldots\right]
$$

This subspace is defined as Krylov-subspace and can be generated without explicitly calculating the moments vectors. A Krylov-subspace has the following Theorem:

Theorem 1 If $\mathcal{Q}$ is orthogonal basis of Krylov-subspace $K_{r}(\mathbf{A}, \mathbf{R}, \mathbf{q})$, then

$$
\mathrm{A} \mathcal{Q}=\mathcal{Q H}
$$


where $H$ is an upper-Hessenberg matrix.

The Arnoldi algorithm is a method to find a set of orthogonal vectors as a basis for a Krylov subspace in (2.12) according to the Theorem 1. The algorithm for a SISO system is shown in Appendix A and Appendix B is for a MIMO system. Arnoldi algorithm has been known to be numerically efficient iterative method and widely used in model-order reduction.

\subsubsection{PRIMA}

PRIMA (passive reduced-order interconnect macromodeling algorithm) [21] is an extension of the Arnoldi algorithm. PRIMA guarantees the passivity of the reducedorder model with simple manipulations of MNA formulation by multiplying -1 for all the inductances and voltages sources in (2.1). PRIMA is an ideal MOR technique for linear passive circuits and have been extended to parametric model order reduction. 


\subsection{Discussion}

Model-order reduction (MOR) has demonstrated great efficiency in simulating largescale systems. Popular reduction algorithms are based on projecting the original large set of equations on a lower dimension space using orthogonal congruence transformation. The most commonly used projection approach for non-parametric systems is based on moment matching which preserves certain number of the leading terms of Taylor series expansion of the response with respect to frequency. To achieve this, a projection matrix is formed using the orthogonal basis of the Krylov subspace spanned by the moment vectors with respect to frequency [1].

In addition, the MOR concept has been extended to include multi-moment matching with respect to frequency and other design parameters such as geometrical layout or substrate characteristics [3]. In the next chapter, the conventional model-order reduction is extended to parametric model-order reduction (PMOR), in which a global basis may be computed for the subspace spanned by the moment vectors associated with frequency as well as the stochastic parameters. 


\section{Chapter 3}

\section{Review of Parametric Model Order Reduction}

\subsection{Introduction}

This chapter reviews the parametric model-order reduction, through which a macromodel of a circuit is generated while preserving the moments with respect to frequency and other electrical parameters. Three prominent methods are reviewed in this chapter. An illustrative example is provided to show the difficulties during their applications for variability analysis.

\subsection{Multi-Dimensional Modeling}

A general linear passive circuit with design of parameters of $\xi=\left[\xi_{1}, \xi_{2}, \xi_{3}, \ldots, \xi_{d}\right]$ can be expressed as

$$
(\boldsymbol{G}(\xi)+s \boldsymbol{C}(\xi)) \mathbf{X}(s, \xi)=\boldsymbol{B U}(s)
$$


The PMOR involves finding a reduced system whose output matches the few leading moments of the original system output $\mathbf{V}(s, \xi)$ in (3.1). In order to find the reduced-order model, first, the block moments of $\mathbf{X}(s, \xi)$ with respect to frequency (denoted by $\mathbf{M}_{s}$ ) are computed. Next, the moments with respect to the random parameters $\xi_{1}, \ldots, \xi_{d}$ (denoted by $\mathbf{M}_{\xi_{1}}, \ldots, \mathbf{M}_{\xi_{d}}$ ), as well as, the cross-derivative block moments (denoted by $\mathbf{M}_{s, \xi_{1}, \ldots, \xi_{d}}$ ) are computed.

In order to compute the block moments $Q_{s}$ or $Q_{\xi}$ of x with respect to frequency and parameters $\xi$, respectively, several scenarios will be considered as follows [2]:

- The block moments of $\boldsymbol{X}$ with respect to frequency(denoted by $Q_{s}$ ) can be computed by Krylov subspace techniques such as PRIMA [21].

- The block moments of $\boldsymbol{X}$ with respect to parameter $\xi_{i}\left(\right.$ denoted by $\left.Q_{\xi_{i}}\right)$ can be computed as [22]:

$$
\begin{aligned}
& Y\left(0, \tilde{\xi}_{i}\right) M_{n}^{\xi_{i}}=-\sum_{r=1}^{m} \frac{\left.\frac{\partial^{r}}{\partial \xi_{i}} Y(0, \xi)\right|_{\xi_{i}=\xi_{o}} M_{n-r}^{\xi_{i}}}{r !} \\
& Y\left(0, \tilde{\xi}_{i}\right) M_{0}^{\xi_{i}}=B .
\end{aligned}
$$

The vector space $Q_{\xi_{i}}$ is formed as:

$$
\operatorname{colspan}\left\{\boldsymbol{Q}_{\boldsymbol{\xi}_{\boldsymbol{i}}}\right\}=\left\{\begin{array}{lllll}
M_{0}^{\xi_{i}} & M_{1}^{\xi_{i}} & M_{2}^{\xi_{i}} & \ldots & M_{m}^{\xi_{i}}
\end{array}\right\}
$$

- In special cases that matrices $\mathrm{C}$ and $\mathrm{G}$ are linear functions with respect to the parameter $\xi_{i}$, techniques such as Arnoldi [23] can be used for better efficiency.

Once all the moments are computed, an orthogonal matrix $\mathbf{Q}$ found whose columns spans the same subspace of the moments [2] is obtained as:

$$
\operatorname{colsp}\{\mathbf{Q}\}=\operatorname{colsp}\left\{\mathbf{M}_{s}, \mathbf{M}_{\xi_{1}}, \ldots, \mathbf{M}_{\xi_{d}}, \mathbf{M}_{s, \xi_{1}, \ldots, \xi_{d}}\right\}
$$


Once the orthogonal matrix is calculated, the ROM can be computed using congruent transformation as follows. Define

$$
\hat{\mathbf{X}}(s, \xi)=\mathbf{Q X}(s, \xi)
$$

where $\hat{\mathbf{X}}(s, \xi) \in \mathbb{R}^{q}$ and $q=q_{s}+q_{s, \xi_{1}, \ldots, \xi_{d}}+\sum_{i=1}^{d} q_{\xi_{i}}$ with $q_{s}, q_{s, \xi_{1}, \ldots, \xi_{d}}$ and $q_{\xi_{i}}$ being the number of columns of $\mathbf{M}_{s}, \mathbf{M}_{s, \xi_{1}, \ldots, \xi_{d}}$ and $\mathbf{M}_{\xi_{i}}$, respectively.

The reduced system can be written as

$$
\begin{aligned}
(\hat{\mathbf{G}}(s, \xi)+s \hat{\mathbf{C}}(s, \xi)) \hat{\mathbf{X}}(s, \xi) & =\hat{\mathbf{B}} \mathbf{U}(s) \\
\hat{\mathbf{V}}(s, \xi) & =\mathbf{L}^{T} \hat{\mathbf{X}}(s, \xi)
\end{aligned}
$$

and in the time-domain, the reduced system can also be represented using

$$
\begin{gathered}
\hat{\mathbf{G}}(\xi) \hat{\mathbf{x}}(t,, \xi)+\hat{\mathbf{C}}(\xi) \frac{d \hat{\mathbf{x}}(t, \xi)}{d t}=\hat{\mathbf{B}} \mathbf{u}(t) \\
\hat{\mathbf{v}}(t, \xi)=\hat{\mathbf{L}}^{T} \hat{\mathbf{x}}(t, \xi)
\end{gathered}
$$

where $\hat{\mathbf{x}}(t, \xi)=\mathbf{Q}^{T} \mathbf{x}(t, \xi), \hat{\mathbf{G}}=\mathbf{Q}^{T} \mathbf{G} \mathbf{Q}, \hat{\mathbf{C}}=\mathbf{Q}^{T} \mathbf{C Q}, \hat{\mathbf{B}}=\mathbf{Q}^{T} \mathbf{B}$ and $\hat{\mathbf{L}}^{T}=\mathbf{L}^{T} \mathbf{Q}$.

This method was widely used in circuit simulation and it is the backbone of parametric model-order reduction. However, the flaw of this method is obvious in that it did not provide an algorithm to compute the moment vectors of the cross terms. To solve this problem, an overview of method in [24] will be presented in the next section. 


\subsection{Explicit Parametric Moment-Matching Model-Order Reduction}

According to the method in [3], the system equation (3.1) will be first transformed to an affine form

$$
\left(\boldsymbol{E}_{\mathbf{0}}+\tilde{\boldsymbol{p}}_{\mathbf{1}} \boldsymbol{E}_{\mathbf{1}}+\tilde{\boldsymbol{p}}_{\mathbf{2}} \boldsymbol{E}_{\mathbf{2}}+\ldots+\tilde{\boldsymbol{p}}_{\boldsymbol{d}} \boldsymbol{E}_{\boldsymbol{d}}\right) X=\boldsymbol{B U}(s)
$$

Where $\tilde{p}_{i}, i=1,2, \ldots d$ are newly defined parameters as some functions (rational, polynomial) of the original parameters $\mathrm{P}$ in equation (3.1). Then response of the system described by (3.9) can be written as

$$
\mathbf{X}(\tilde{p})=\left(\boldsymbol{I}-\sum_{i=1}^{d} \tilde{p}_{i} \tilde{\boldsymbol{A}}_{\boldsymbol{i}}\right)^{-1} \tilde{\boldsymbol{R}} \boldsymbol{U}(s)
$$

where

$$
\begin{aligned}
& \tilde{\boldsymbol{p}}=\left(\tilde{\boldsymbol{p}}_{\mathbf{1}}, \tilde{\boldsymbol{p}}_{\mathbf{2}}, \ldots, \tilde{\boldsymbol{p}}_{\boldsymbol{d}}\right)^{\boldsymbol{T}}, \\
& \tilde{\boldsymbol{A}}_{i}=-\boldsymbol{E}_{0}^{-1} \boldsymbol{E}_{i}, \\
& \tilde{\boldsymbol{R}}=\boldsymbol{E}_{0}^{-1} \boldsymbol{B} .
\end{aligned}
$$

The Taylor expansion of at expansion point $\tilde{p}=\left(p_{1}, p_{2}, \ldots, p_{d}\right)^{T}$ will be

$$
\mathrm{X}(\tilde{p})=\sum_{\rho=0}^{\infty}\left(\sum_{i=1}^{d} \Delta \tilde{p}_{i} \tilde{A}_{i}\right)^{\rho} \tilde{R}
$$

where $\Delta \tilde{p}_{i}=p_{i}-\tilde{p}_{i}$.

The projection operator $\mathbf{Q}$ can be explicitly generated such that its column are 
the moment vectors of the parametric system (3.12) as

$$
\begin{aligned}
& \operatorname{colspan}\{\boldsymbol{Q}\}=\begin{array}{r}
\operatorname{span}\left\{\tilde{\boldsymbol{R}}, \tilde{\boldsymbol{A}_{1}} \tilde{\boldsymbol{R}}, \tilde{\boldsymbol{A}}_{2} \tilde{\boldsymbol{R}}, \ldots, \tilde{\boldsymbol{A}}_{p} \tilde{\boldsymbol{R}}, \tilde{\boldsymbol{A}}_{1}^{2} \tilde{\boldsymbol{R}}, \tilde{\boldsymbol{A}}_{1} \tilde{\boldsymbol{A}_{2}} \tilde{\boldsymbol{R}}, \ldots\right. \\
\left.\tilde{\boldsymbol{A}}_{1} \tilde{\boldsymbol{A}_{d}} \tilde{\boldsymbol{R}}, \tilde{\boldsymbol{A}}_{2}^{2} \tilde{\boldsymbol{R}}, \tilde{\boldsymbol{A}}_{2} \tilde{\boldsymbol{A}}_{\mathbf{1}} \tilde{\boldsymbol{R}}, \ldots\right\}
\end{array}
\end{aligned}
$$

It is noted that the computational cost is mainly one $\mathrm{LU}$ decomposition on $\mathbf{E}_{\mathbf{0}}$ to generate each columns of the subspace explicitly, and then apply QR decomposition to generate the orthogonal basis of this subspace. Once the subspace $\mathbf{Q}$ is formulated, the dynamic system (3.9) can be reduced by congruent transformation as

$$
\left(\tilde{\boldsymbol{E}}_{\mathbf{0}}+\tilde{\boldsymbol{p}}_{\mathbf{1}} \tilde{\boldsymbol{E}}_{\mathbf{1}}+\tilde{\boldsymbol{p}}_{\mathbf{2}} \tilde{\boldsymbol{E}}_{\mathbf{2}}+\ldots+\tilde{\boldsymbol{p}}_{\boldsymbol{d}} \tilde{\boldsymbol{E}}_{\boldsymbol{d}}\right) \tilde{\boldsymbol{X}}=\tilde{\boldsymbol{B}} \boldsymbol{U}(s),
$$

where $\tilde{\mathbf{E}}_{\mathbf{i}}=\mathbf{Q}^{\mathbf{t}} \mathbf{E Q}$.

This macro-model preserving the dynamic information of both frequency and parameters of system (3.1) can be now simulated in both frequency domain and time domain.

The major disadvantage of the above method is that, generation of the projection operator Q explicitly will cause numerical instability. Similar to AWE, the calculation of moment vectors converges rapidly to an eigenvector of $A_{i}$ associated with the eigenvalue of largest magnitude [4]. To avoid this problem, an overview of a robust implicit moment matching algorithm [4] is reviewed in the next section. 


\subsection{Implicit Parametric Model-Order Reduction}

According to the method in [4], the implicit moment matching technique is based on the recursive relations of power series of $\mathbf{X}$ in equation (3.12). The power series of $\mathbf{X}$ can be written as

$$
\begin{aligned}
\mathbf{X}(\tilde{p})=\sum_{\rho=0}^{\infty} \mathbf{A}^{\rho} \tilde{\mathbf{R}}=\tilde{\mathbf{R}}+\mathbf{A} \tilde{\mathbf{R}}+\mathbf{A}^{2} \tilde{\mathbf{R}}+ & \ldots+\mathbf{A}^{\rho} \tilde{\mathbf{R}} \\
& =\mathbf{x}_{\mathbf{0}}+\mathbf{x}_{\mathbf{1}}+\mathbf{x}_{\mathbf{2}}+\mathbf{x}_{\mathbf{3}}+\ldots+\mathbf{x}_{\rho}+\ldots
\end{aligned}
$$

by defining

$$
\begin{aligned}
\mathbf{A} & =\sum_{i=1}^{d} \Delta p_{i} \tilde{\mathbf{A}}_{\mathbf{i}} \\
\mathbf{x}_{\mathbf{0}} & =\tilde{\mathbf{R}} \\
\mathbf{x}_{\mathbf{1}} & =\mathbf{A} \tilde{\mathbf{R}} \\
\mathbf{x}_{\mathbf{2}} & =\mathbf{A}^{2} \tilde{\mathbf{R}} \\
& \ldots \\
\mathbf{x}_{\rho} & =\mathbf{A}^{\rho} \tilde{\mathbf{R}}
\end{aligned}
$$

and $\mathbf{x}_{\mathbf{i}}, i=0,1,2, \ldots, \rho$ are called as $i^{\text {th }}$ order Taylor expansion of $\mathbf{X}$.

It is clear that $\mathbf{x}_{\mathbf{i}}$ has the recursive relations as $\mathbf{x}_{\mathbf{i}}=\mathbf{A} \mathbf{x}_{\mathbf{i}-\mathbf{1}}$. Define $\mathbf{M}_{\mathbf{i}}$ as the moment vector space of $i^{\text {th }}$ order, the moment vector space $\mathbf{M}_{\mathbf{i}}$ from order 
$i=0,1,2,3, \ldots, \rho$ will also have the recursive relations as

$$
\begin{aligned}
\mathbf{M}_{\mathbf{0}} & =[\tilde{\mathbf{R}}] \\
\mathbf{M}_{\mathbf{1}} & =\left[\tilde{\mathbf{A}}_{\mathbf{1}} \mathbf{M}_{\mathbf{0}}, \tilde{\mathbf{A}}_{\mathbf{2}} \mathbf{M}_{\mathbf{0}}, \ldots, \tilde{\mathbf{A}}_{\mathbf{d}} \mathbf{M}_{\mathbf{0}}\right] \\
\mathbf{M}_{\mathbf{2}} & =\left[\tilde{\mathbf{A}}_{\mathbf{1}} \mathbf{M}_{\mathbf{1}}, \tilde{\mathbf{A}}_{\mathbf{2}} \mathbf{M}_{\mathbf{1}}, \ldots, \tilde{\mathbf{A}}_{\mathbf{d}} \mathbf{M}_{\mathbf{1}}\right] \\
& \ldots \\
\mathbf{M}_{\rho} & =\left[\tilde{\mathbf{A}}_{\mathbf{1}} \mathbf{M}_{\rho-1}, \tilde{\mathbf{A}}_{\mathbf{2}} \mathbf{M}_{\rho-1}, \ldots, \tilde{\mathbf{A}}_{\mathbf{d}} \mathbf{M} \rho-\mathbf{1}\right],
\end{aligned}
$$

Using the recursive relations of $\mathbf{M}_{\mathbf{i}}$ in (3.17), the implicit recursive modified GramSchmidt(MGS) process $[4,25]$ is used to construct the projection operator $\mathbf{Q}$ with numerically stable guaranteed and the pseudo code for generating the projection operator $\mathbf{Q}$ can be found in [25]. 


\subsection{Applications using Parametric Model-Order Reduction}

In order to show the difficulties of conventional PMOR approach, this section will present the simulation results based on implicit conventional PMOR [4] comparing with those obtained from the Monte Carlo based simulations.

In this example, we consider the 4 coupled interconnect circuits shown in Fig. 3.1. The lines are modeled using 100 lumped segments. The circuit is excited by a current source attached to the near end of the first line. Five stochastic physical parameters were considered and their nominal values are shown in the figure.

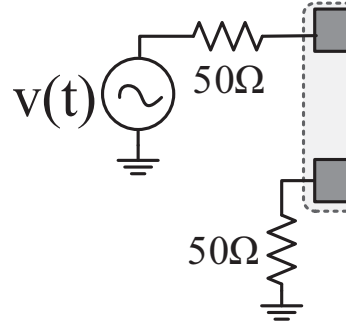

$$
\begin{aligned}
& w=0.5 \mathrm{~mm} \\
& d=2 \mathrm{~mm} \\
& h=2 \mathrm{~mm} \\
& \text { Conductivity: } \\
& \sigma=5.6 \mathrm{E} 7 \mathrm{~S} / \mathrm{m} \\
& \text { Relative Permittivity: } \\
& \varepsilon_{r}=4.5
\end{aligned}
$$

Fig. 3.1: Four coupled interconnects circuit

Fig. 3.2 shows the mean and $\pm 3 \sigma$ tolerances using conventional PMOR comparing with the results from original system. The results from PMOR match reasonably well with original system. The related CPU cost is shown in Table 3.1. As seen, the conventional PMOR failed to yield significant speed-up compared to using the original system. 


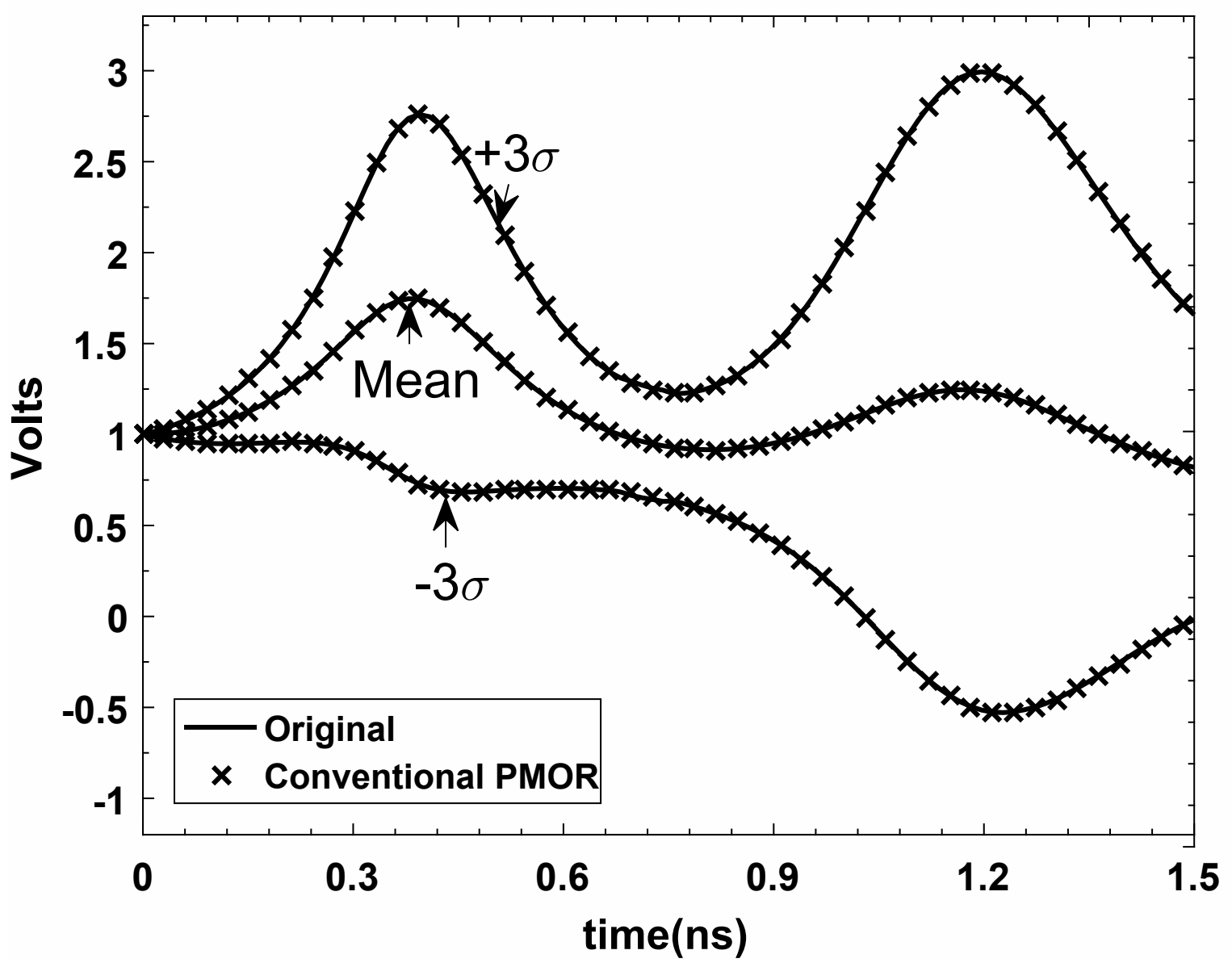

Fig. 3.2: Statistical analysis for the magnitude of the far-end voltage of active line(Example in Section 3.5)

Table 3.1: Problem Sizes and CPU computation time for Conventional PMOR

\begin{tabular}{||c|c|c||}
\hline & Size & CPU cost \\
\hline \hline Original System & 1224 by 1224 & $147.5 \mathrm{sec}$ \\
\hline Reduced System & 106 by 106 & $108.5 \mathrm{sec}$ \\
\hline
\end{tabular}




\subsection{Difficulties with Conventional PMOR}

Comparing the above methods, method in [2] provides an implicit moments matching PMOR algorithm by which the reduced macro-model preserves the passivity. Also because it neglected the moments corresponding to the cross-derivative terms, the size of the reduced macro-model is relatively small, comparing to the other methods. However the limitation of this algorithm is obvious in that it cannot cover universal cases.

Method in [3] addresses the limitations of [2]. But this algorithm is numerically unstable, which the circuit simulations always wish to avoid.

Method in [4] provides a numerically stable algorithm to generate a macro-model with all the expansion of parameters. However, typically the order of moments required for electrical parameters is relatively low, but the order of moments with respect to frequency is much higher. This will cause the macro-model to preserve the order of moments in each dimension and hence, the size of the macro-model grows rapidly when the number of parameters increases. Example in 3.1 further illustrated that this algorithm does not yield significant CPU speed-up for variability analysis.

A common goal shared between all these methods [2-4] is to obtain a dynamic reduced macro-model by preserving the moments of expansions on frequency and parameters, when forming the moments subspace. One obvious drawback of the global basis approach is that the size of the reduced-order model is in general, relatively large leading to small computational savings over solving the original full system. This is against the goal of variability analysis where the objective is to obtain a parameterized reduced model that can be used to efficiently compute the statistical properties of the system response.

To address the above difficulties, in the rest of this thesis, a new parameterized 
model-order reduction (PMOR) algorithm that poses the variability analysis problem from a different perspective is presented. 


\section{Chapter 4}

\section{Proposed Variability Analysis using PMOR in Frequency Domain}

In Chapter 3, several methods of conventional PMOR were reviewed and also discussed the difficulties while applying the conventional PMOR for variability analysis. Addressing these issues, a new parameterized model-order reduction (PMOR) algorithm is described in Section 4.2 to 4.4. Two examples are presented in Section 4.5.

\subsection{Concept Associated with Proposed PMOR}

The key factor that is associated with the development of the proposed method is that, the response of circuits is a highly nonlinear function w.r.t. frequency. Thus, a large number of Taylor-series moments is required to accurately approximate the transfer function over the frequency band-width of interest.

This can be illustrated through a practical interconnect example (for details see Example 1 in Numerical Example Section 4.5 with 5 stochastic parameters). Fig. 4.1 and Fig. 4.2 show the output waveform at the far-end of the active-line \#1 and victim line \#2 with respect to both frequency and the width of the transmission lines. As seen, the output waveforms with respect to frequency are highly nonlinear, 
which would require large number of block moments with respect to frequency for a reasonable approximation, leading to a large reduced-order model. Fig. 4.3 and Fig. 4.4 show the responses at the far-end of the active-line \#1 and victim line \#2 at a fixed frequency with respect to width and separation between the lines. As can be seen, the responses with respect to stochastic parameters are relatively smooth and hence require only few moments for capturing the corresponding stochastic behaviors. Thus, by eliminating the block moments with respect to frequency, the size of the resulting reduced-order model can be kept very small.

Taking advantage of the above observation, instead of creating a macro-model covering the whole frequency band, the proposed algorithm is based on the idea of generating several local models at the selected frequencies of interest by computing an orthogonal matrix $\mathcal{Q}$ through multi-directional moment matching in the parameter space only.

Excluding the moments with respect to frequency leads to much smaller reducedmodels since in general, because the variation of circuit response with respect to stochastic parameters is much smoother than the corresponding variation with frequency. In addition, the local models preserve the stochastic parameters as symbolic quantities. As a result, a change in parameters does not require computing new reduced-order models, but simply the evaluation of reduced-order models for the new parameter values. Consequently, the stochastic response of the circuit can be obtained by simulating the local reduced-order models instead of the original large system leading to significant reduction in the computational cost.

Assuming that frequency vector $\boldsymbol{f}=\left[f_{1}, f_{2}, \ldots, f_{i}, \ldots\right]$ has been optimized using commercial program such as HSPICE or PSPICE, this chapter will start from single parameter case, then expand to two-parameter case and subsequently provide an algorithm for the general case using both explicit and implicit moments matching 


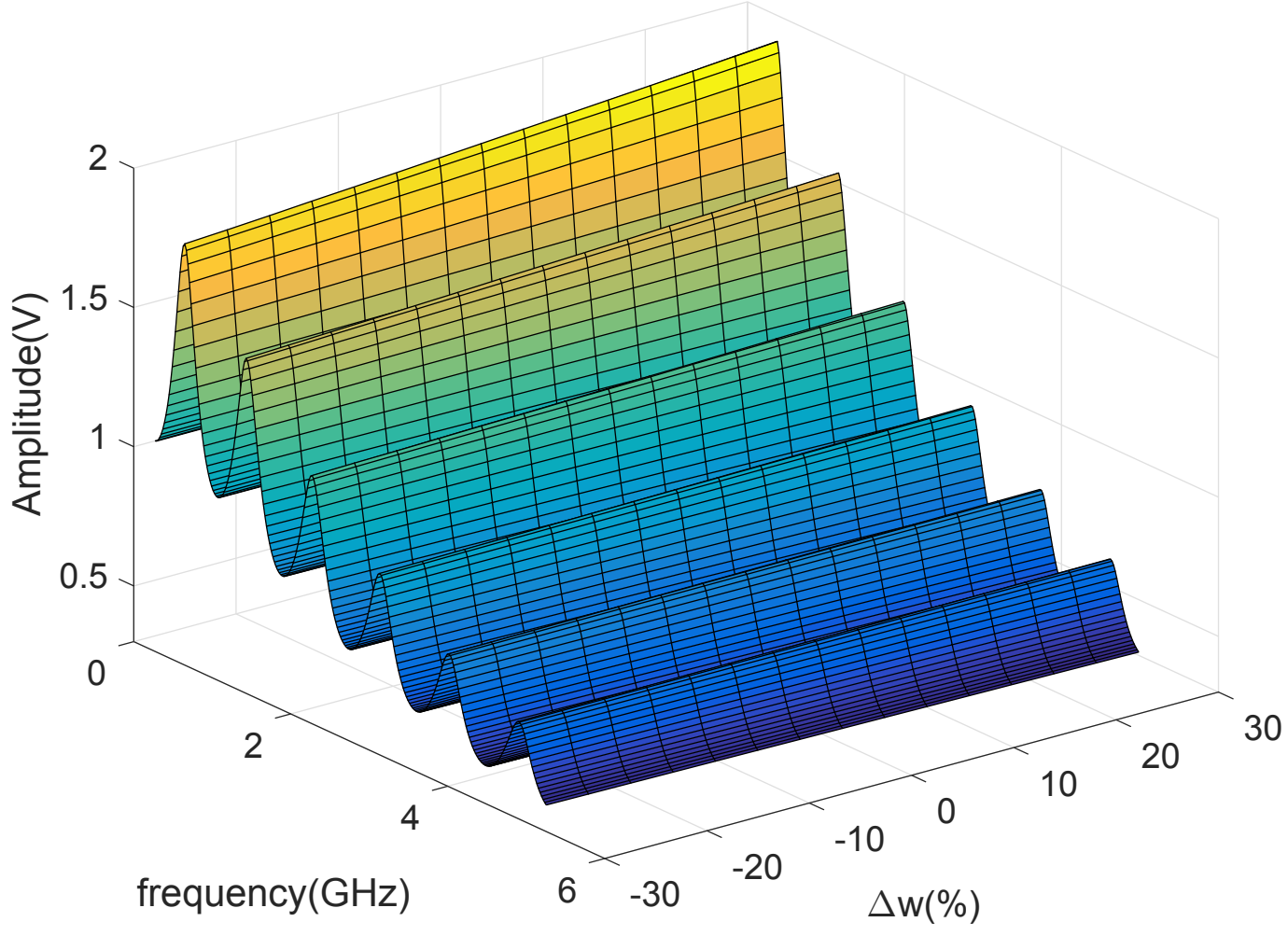

Fig. 4.1: Response of active line of an interconnect circuit w.r.t. frequency and width of lines

techniques. 


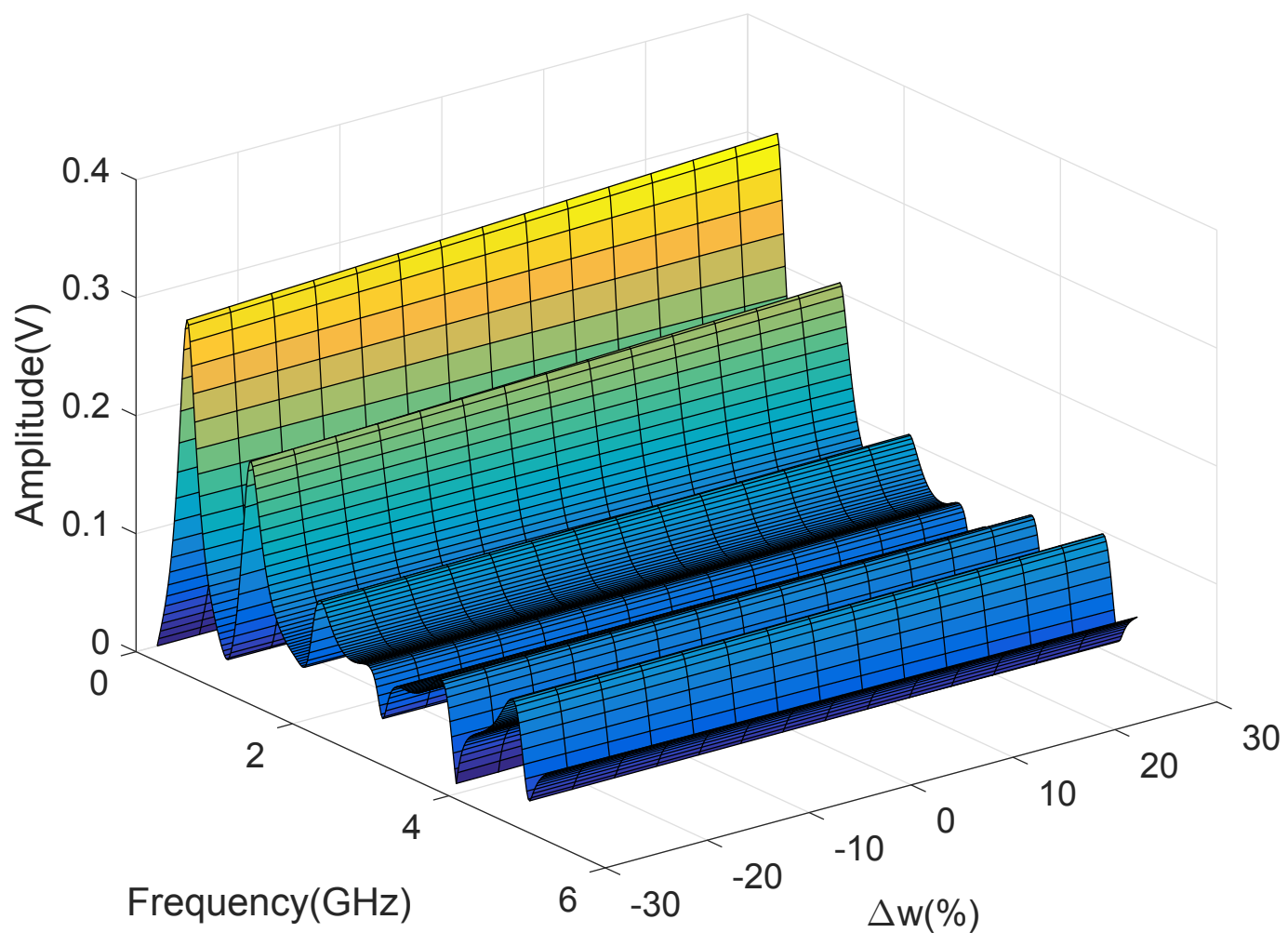

Fig. 4.2: Response of victim line of an interconnect circuit w.r.t. frequency and width of lines 


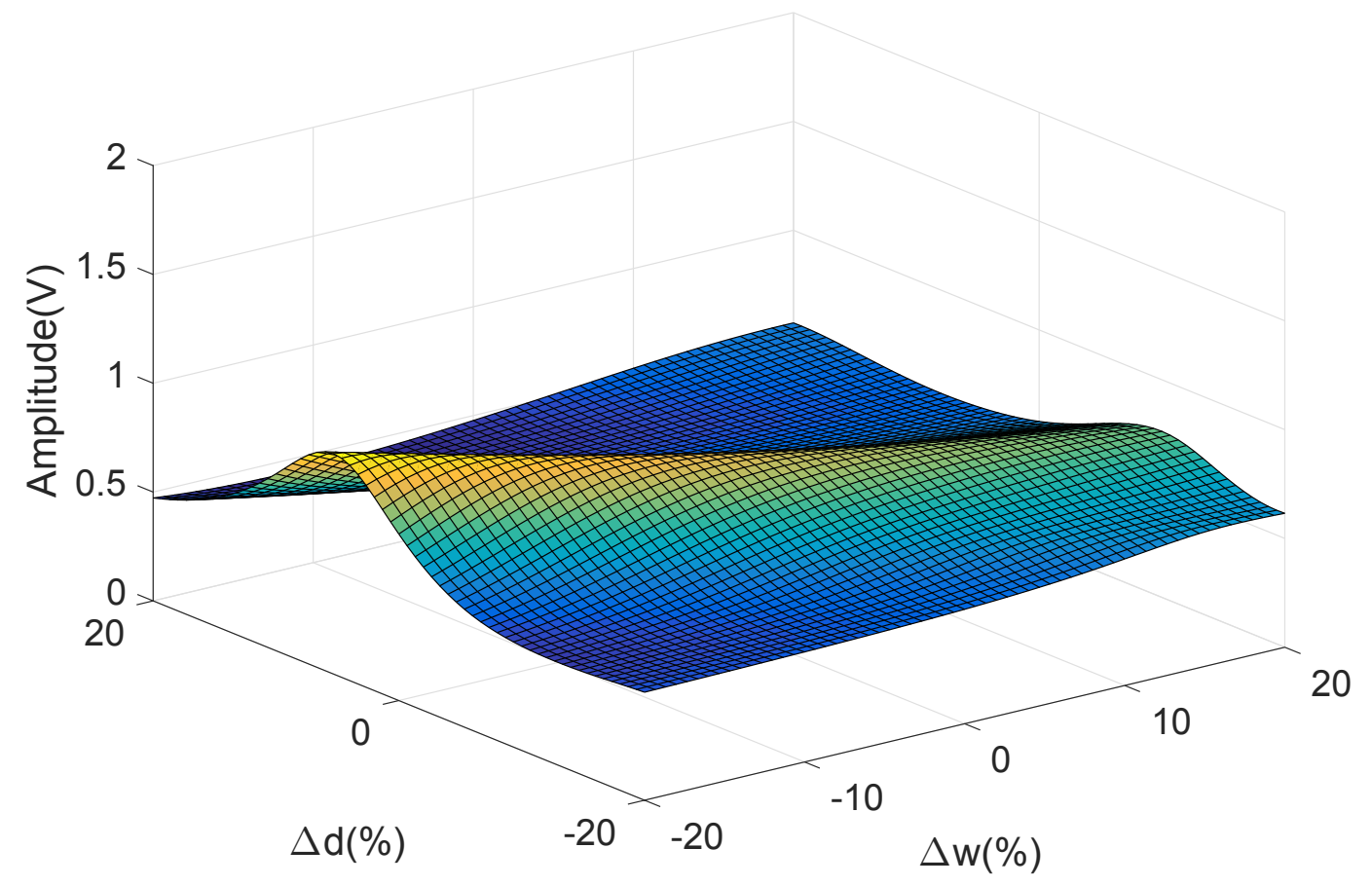

Fig. 4.3: Response of active line of an interconnect circuit at $3 \mathrm{GHz}$ w.r.t. width of lines and separations between lines. 


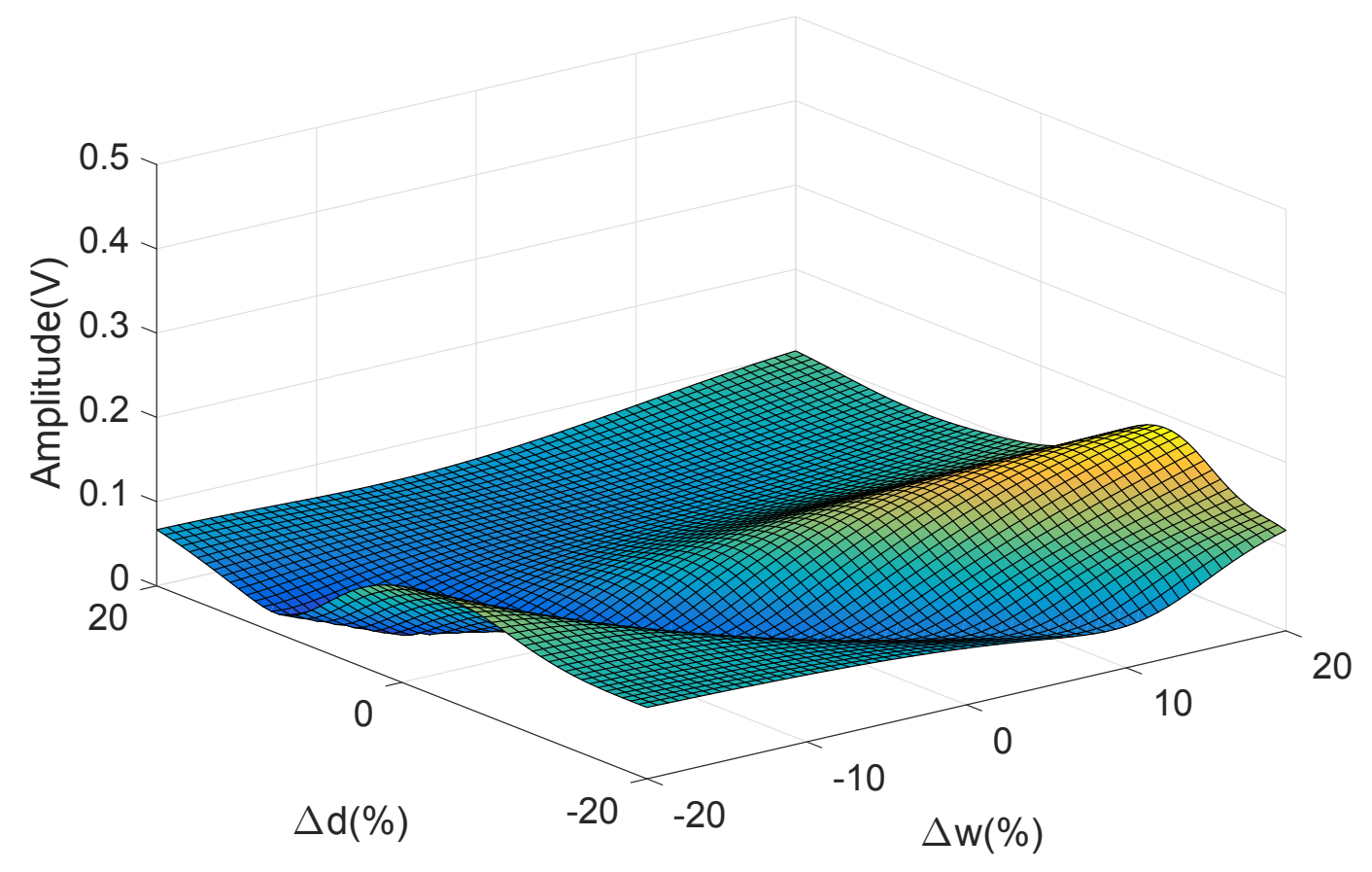

Fig. 4.4: Response of victim line of an interconnect circuit at $3 \mathrm{GHz}$ w.r.t. width of lines and separations between lines. 


\subsection{Case of Single Random Parameter}

In this section, the simple case of single random parameter is considered to outline the steps associated with the proposed PMOR algorithm. Consider the Modified Nodal Analysis(MNA) equations of a linear circuit including random parameters at a fixed frequency $s$ as

$$
\begin{array}{r}
(\mathbf{G}(\xi)+\mathbf{s} \mathbf{C}(\xi)) \mathbf{X}(s, \xi)=\mathbf{B U}(s) \\
\mathbf{V}(s, \xi)=\mathbf{L}^{T} \mathbf{X}(s, \xi)
\end{array}
$$

where $\mathbf{G}, \mathbf{C} \in \mathbb{R}^{N \times N}$ are functions of the parameters $\xi$ describing the memoryless and memory elements in the circuit, respectively, $\mathbf{X}(s) \in \mathbb{R}^{N}$ is the vector of unknown node voltages and currents, $\mathbf{B} \in \mathbb{R}^{N}$ is the vector of independent stimuli to the circuit, $N$ is the number of unknown voltages/currents in the circuit, $\xi$ is a random variable and $s$ is the Laplace variable which is fixed.

Assuming that $\mathbf{G}(\xi)$ and $\mathbf{C}(\xi)$ are linear function of $\xi$ as

$$
\begin{aligned}
& \mathbf{G}(\xi)=\mathbf{G}_{0}+\xi \mathbf{G}_{1} \\
& \mathbf{C}(\xi)=\mathbf{C}_{0}+\xi \mathbf{C}_{1}
\end{aligned}
$$

equation (4.1) can be written as

$$
\left(\left(\mathbf{G}_{0}+s \mathbf{C}_{0}\right)+\xi\left(\mathbf{G}_{1}+s \mathbf{C}_{1}\right)\right) \mathbf{X}(s, \xi)=\mathbf{B U}(s)
$$

where $\mathbf{G}_{0}$ and $\mathbf{C}_{0}$ are the nominal values of $\mathbf{G}$ and $\mathbf{C}, \xi$ is a random variable and $\mathbf{G}_{0}$ and $\mathbf{C}_{0}$ are the elements effected by random variable $\xi$. Since frequency $\mathrm{s}$ is fixed, equation (4.3) is an algebraic function instead of a dynamic function. By denoting $\mathbf{E}_{0}=\left(\mathbf{G}_{0}+s \mathbf{C}_{0}\right)$ and $\mathbf{E}_{1}=\left(\mathbf{G}_{1}+s \mathbf{C}_{1}\right)$ and dropping $\mathrm{s}$ in $\mathbf{X}(s, \xi)$ and $\mathbf{U}(s)$, the 
algebraic function becomes

$$
\left(\mathbf{E}_{0}+\xi \mathbf{E}_{1}\right) \mathbf{X}(\xi)=\mathbf{B U}
$$

and the response $\mathbf{X}$ is a function of parameter $\xi$

$$
\begin{aligned}
\mathbf{X}(\xi) & =\left(\mathbf{E}_{0}+\xi \mathbf{E}_{1}\right)^{-1} \mathbf{B U} \\
& =\left(\mathbf{I}-\xi\left(-\mathbf{E}_{0}^{-1} \mathbf{E}_{1}\right)\right)^{-1} \mathbf{E}_{0}^{-1} \mathbf{B} \mathbf{U} \\
& =(\mathbf{I}-\xi \mathcal{A})^{-1} \boldsymbol{R} \mathbf{U}
\end{aligned}
$$

where $\mathcal{A}=-\mathbf{E}_{0}^{-1} \mathbf{E}_{1}$ and $\mathcal{R}=\mathbf{E}_{0}^{-1} \mathbf{B}$.

To obtain a reduced-order model of (4.4), the projection operator $\mathcal{Q}$ must be generated that columns of the vector space are the moments of response spanned by parameter $\xi$. The response of $\mathbf{X}$ can be expanded into a Taylor series at expansion point $\xi=0$ as below,

$$
\mathbf{X}(s, \xi)=\sum_{\rho=0}(\xi \mathcal{A})^{\rho} \mathbf{R U}
$$

and the columns of moments spanned by parameter $\xi$ of the output function in (4.5) span the following subspace

$$
\mathcal{S}=\operatorname{colspan}\left\{\mathbf{R}, \mathcal{A R}, \mathcal{A}^{2} \mathbf{R}, \ldots, \mathcal{A}^{\rho} \mathbf{R}, \ldots\right\}
$$

This is a Krylov space and it satisfies the following property of the Krylov subspace:

$$
\mathcal{A Q}=\mathcal{Q H}
$$

where columns of $\mathcal{Q}$ is the orthogonal basis of $\mathcal{S}$ and $\mathcal{H}$ is an upper-Hessenberg Matrix whose $\mathcal{H}(i, j)=0$ for $i>j+1$. Arnoldi algorithm [26] can be applied to 
solve equation (4.8) and construct the projection matrix $\mathcal{Q}$.

Once the projection matrix operator $\mathcal{Q}$ is constructed, a reduced-order model of (4.5) can be obtained using congruent transformation as

$$
\left(\tilde{\mathbf{E}}_{0}+\xi \tilde{\mathbf{E}}_{1}\right) \tilde{\mathbf{X}}(\xi)=\tilde{\mathbf{B}} \mathbf{U}
$$

where

$$
\begin{aligned}
\tilde{\mathbf{E}}_{0} & =\mathcal{Q}^{T} \mathbf{E}_{0} \mathcal{Q} \\
\tilde{\mathbf{E}}_{1} & =\mathcal{Q}^{T} \mathbf{E}_{1} \mathcal{Q} \\
\tilde{\mathbf{B}} & =\mathcal{Q}^{T} \mathbf{B} .
\end{aligned}
$$

Now the original system in dimension $N$ is projected to a lower dimension $m$ such that $\tilde{\mathbf{X}}(s) \in \mathbb{R}^{m}$ is the unknowns of the reduced system. Then the output of interest can be calculated as:

$$
\begin{aligned}
\tilde{\mathbf{X}}(\xi) & =\left(\tilde{\mathbf{E}}_{0}+\xi \tilde{\mathbf{E}}_{1}\right)^{-1} \tilde{\mathbf{B}} \mathbf{U} \\
\mathbf{V}(\xi) & =\tilde{\mathbf{L}}^{T} \tilde{\mathbf{X}}(\xi),
\end{aligned}
$$

where

$$
\tilde{\mathbf{L}}^{T}=\mathbf{L}^{T} \mathcal{Q}
$$

Then the variability analysis at this frequency point can be obtained using the reduced-order model.

To summarize, assuming that a frequency vector $\boldsymbol{f}=\left[f_{1}, f_{2}, \ldots, f_{i}\right]$ is generated, a pseudo-code for single parameter case is given in Algorithm 1 and the steps of the Arnoldi algorithm in function $[\mathcal{Q}, \mathcal{H}]=\operatorname{Arnoldi} A \operatorname{Algorithm}(\mathcal{A}, \mathcal{R}, m)$ is given in the Appendix A.

In the next section, the expansion of the idea to generate local models at selective 


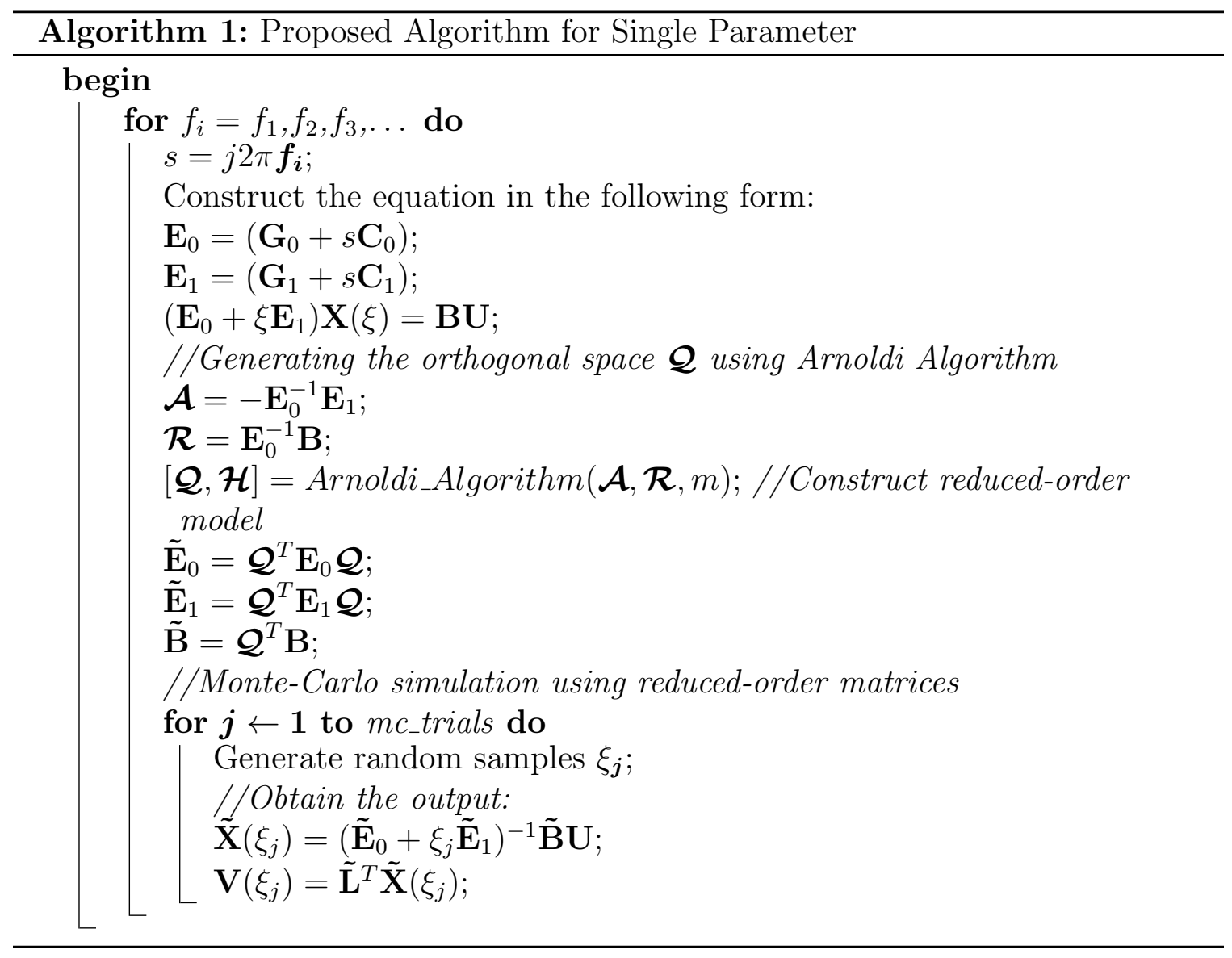


frequencies with more random variables using explicit moment-matching techniques is presented. 


\subsection{Case of Multiple Parameters}

Given that $\mathbf{G}(\xi)$ and $\mathbf{C}(\xi)$ are functions of $\xi$, where $\xi$ is a n-dimensional vector collecting all the random circuit parameters, the parametrized MNA equation is

$$
\begin{array}{r}
\left(\mathbf{G}\left(\xi_{\mathbf{1}}, \xi_{\mathbf{2}}, \xi_{\mathbf{3}}, \ldots, \xi_{\mathbf{n}}\right)+\mathbf{s} \mathbf{C}\left(\xi_{1}, \xi_{2}, \xi_{3}, \ldots, \xi_{n}\right)\right) \mathbf{X}(s, \xi)=\mathbf{B U}(s) \\
\mathbf{V}(s, \xi)=\mathbf{L}^{T} \mathbf{X}(s, \xi)
\end{array}
$$

In order to apply the proposed method, MNA equations of (4.13) will be transformed into an affine form. Since the proposed method is based on fixing the frequency, a function of parameter $\xi$ can be defined as

$$
\mathbf{E}(\xi)=\mathbf{G}\left(\xi_{1}, \xi_{2}, \xi_{3}, \ldots, \xi_{n}\right)+\mathbf{s} \mathbf{C}\left(\xi_{1}, \xi_{2}, \xi_{3}, \ldots, \xi_{n}\right)
$$

To form the function $\mathbf{E}(\xi)$ into the affine form, $\mathbf{E}(\xi)$ is expanded into Taylor series

at expansion point where all the parameters are at their nominal values $\hat{\xi}$. Using this idea, the function $\mathbf{E}(\xi)$ can be written as:

$$
\begin{aligned}
& \mathbf{E}(\xi)=\mathbf{E}_{\mathbf{o}}+\sum_{i=1}^{n} \tilde{\xi}_{i} \mathbf{E}_{i}+\sum_{i, j=1}^{n} \tilde{\xi}_{i} \tilde{\xi}_{j} \mathbf{E}_{i, j}+\sum_{i, j, k=1}^{n} \tilde{\xi}_{i} \tilde{\xi}_{j} \tilde{\xi}_{k} \mathbf{E}_{i, j, k} \\
& +\cdots+\sum_{i, j, k, \cdots, l=1}^{n} \underbrace{\tilde{\xi}_{i} \tilde{\xi}_{j} \tilde{\xi}_{k} \ldots \tilde{\xi}_{l}}_{\eta \text { terms }} \mathbf{E}_{i, j, k, \ldots, l}+\ldots,
\end{aligned}
$$

where $\tilde{\xi}_{i}=\xi_{i}-\hat{\xi}_{i}, i=1,2,3, \ldots, n$ and $\underbrace{\mathbf{E}_{i, j, k, l}, \ldots, l}_{\eta \text { terms }}=\frac{1}{\eta !} \frac{\partial^{\eta} \mathbf{E}\left(\hat{\xi}_{1}, \hat{\xi}_{\mathbf{2}}, \hat{\xi}_{\mathbf{3}}, \ldots, \hat{\xi}_{\mathbf{n}}\right)}{\partial \xi_{1} \partial \xi_{2} \partial \xi_{3} \ldots \partial \xi_{\eta}}$.

The proposed method is not limited by the fact that the affine form of function E must be Taylor series. The proposed method can be applied as long as function $\mathbf{E}$ 
can be transformed into the affine form as

$$
\mathbf{E}(\xi)=\mathbf{E}_{0}+\zeta_{1} \mathbf{E}_{1}+\zeta_{2} \mathbf{E}_{2}+\cdots+\zeta_{d} \mathbf{E}_{d}
$$

$\zeta$ are new defined parameters which are some functions of the original parameter $\xi$, $\mathbf{E}_{i}, i=1,2,3, \ldots, d$ are constant. Then the MNA equation becomes

$$
\left(\mathbf{E}_{0}+\zeta_{1} \mathbf{E}_{1}+\zeta_{2} \mathbf{E}_{2}+\cdots+\zeta_{d} \mathbf{E}_{d}\right) \mathbf{X}=\mathbf{B U}
$$

In order to generate the moment vectors of response $\mathbf{X}$ expanded by the new parameters $\zeta$, the output function of $\mathbf{X}$ can be written as:

$$
\begin{aligned}
\mathbf{X} & =\left(\mathbf{E}_{\mathbf{0}}+\zeta_{1} \mathbf{E}_{\mathbf{1}}+\zeta_{2} \mathbf{E}_{\mathbf{2}}+\zeta_{3} \mathbf{E}_{\mathbf{3}}+\cdots+\zeta_{d} \mathbf{E}_{\mathbf{d}}\right)^{-1} \mathbf{B U} \\
& =\left(\mathbf{I}-\zeta_{1}\left(-\mathbf{E}_{0}^{-1} \mathbf{E}_{1}\right)-\zeta_{2}\left(-\mathbf{E}_{0}^{-1} \mathbf{E}_{2}\right)-\cdots-\zeta_{d}\left(-\mathbf{E}_{0}^{-1} \mathbf{E}_{d}\right)\right)^{-1} \mathbf{E}_{0}^{-1} \mathbf{B} \mathbf{U}
\end{aligned}
$$

By defining $\mathcal{A}_{i}=-\mathbf{E}_{0}^{-1} \mathbf{E}_{i}, i=1,2,3, \ldots, d$ and $\mathcal{R}=\mathbf{E}_{0}^{-1} \mathbf{B}$, the output function becomes

$$
\begin{aligned}
\mathbf{X} & =\left(\mathbf{I}-\zeta_{1} \mathcal{A}_{1}-\zeta_{2} \mathcal{A}_{2}-\zeta_{3} \mathcal{A}_{3}-\cdots-\zeta_{d} \mathcal{A}_{d}\right)^{-1} \mathcal{R} \mathbf{U} \\
& =\left(\mathbf{I}-\left(\sum_{i=1}^{d} \zeta_{i} \mathcal{A}_{i}\right)\right)^{-1} \mathcal{R} \mathbf{U} .
\end{aligned}
$$


Then the output function is transformed into a power series as:

$$
\begin{aligned}
\mathbf{X}=\sum_{k=0}\left(\sum_{i=1}^{d} \zeta_{i} \mathcal{A}_{i}\right)^{k} \mathcal{R} \mathbf{U} \\
=\mathcal{R} \mathbf{U}+\sum_{k=1}\left(\zeta_{1} \mathcal{A}_{1}+\zeta_{2} \mathcal{A}_{2}+\cdots+\zeta_{d} \mathcal{A}_{d}\right)^{k} \mathcal{R} \mathbf{U} \\
=\mathcal{R} \mathbf{U}+\left(\zeta_{1} \mathcal{A}_{1}+\zeta_{2} \mathcal{A}_{2}+\cdots+\zeta_{d} \mathcal{A}_{d}\right) \mathcal{R} \mathbf{U} \\
\quad+\left(\zeta_{1} \mathcal{A}_{1}+\zeta_{2} \mathcal{A}_{2}+\cdots+\zeta_{d} \mathcal{A}_{d}\right)^{2} \mathcal{R} \mathbf{U} \\
\quad+\left(\zeta_{1} \mathcal{A}_{1}+\zeta_{2} \mathcal{A}_{2}+\cdots+\zeta_{d} \mathcal{A}_{d}\right)^{3} \mathcal{R} \mathbf{U} \\
\quad \vdots \\
\quad+\left(\zeta_{1} \mathcal{A}_{1}+\zeta_{2} \mathcal{A}_{2}+\cdots+\zeta_{d} \mathcal{A}_{d}\right)^{k} \mathcal{R} \mathbf{U}
\end{aligned}
$$

In order to construct the orthogonal projection operator $\mathcal{Q}$, a subspace $\mathcal{S}$ can be generated explicitly so that its columns match the leading $\mathrm{n}$ columns moments of the original system response (4.18) as

$$
\begin{aligned}
& \mathcal{S}=\operatorname{colspan}\left\{\mathbf{R}, \mathcal{A}_{1} \mathbf{R}, \mathcal{A}_{2} \mathbf{R}, \mathcal{A}_{3} \mathbf{R}, \ldots, \mathcal{A}_{d} \mathbf{R}\right. \\
& \mathcal{A}_{1}^{2} \mathbf{R}, \mathcal{A}_{1} \mathcal{A}_{2} \mathbf{R}, \mathcal{A}_{1} \mathcal{A}_{3} \mathbf{R}, \ldots, \mathcal{A}_{1} \mathcal{A}_{d} \mathbf{R} \\
&\left.\mathcal{A}_{2} \mathcal{A}_{1} \mathbf{R}, \mathcal{A}_{2}^{2} \mathbf{R}, \mathcal{A}_{2} \mathcal{A}_{3} \mathbf{R}, \ldots, \mathcal{A}_{2} \mathcal{A}_{d} \mathbf{R}, \ldots\right\}
\end{aligned}
$$

When subspace $\mathcal{S}$ is constructed, the orthogonal basis $\mathcal{Q}$ of the subspace $\mathcal{S}$ can be obtained using QR algorithm. The reduced-order model as

$$
\left(\tilde{\mathbf{E}}_{\mathbf{0}}+\zeta_{1} \tilde{\mathbf{E}}_{\mathbf{1}}+\zeta_{2} \tilde{\mathbf{E}}_{\mathbf{2}}+\ldots+\zeta_{d} \tilde{\mathbf{E}}_{\mathbf{d}}\right) \tilde{\mathbf{X}}=\tilde{\mathbf{B}} \mathbf{U}
$$


can be produced by a congruent transformation as

$$
\begin{aligned}
\tilde{\mathbf{E}}_{i} & =\mathcal{Q}^{T} \mathbf{E}_{i} \mathcal{Q} \\
\tilde{\mathbf{B}} & =\mathcal{Q}^{T} \mathbf{B} .
\end{aligned}
$$

It is to be noticed that size of the system is reduced from $\mathbf{E}_{\mathbf{i}} \in \mathbb{R}^{N \times N}$ to $\tilde{\mathbf{E}}_{\mathbf{i}} \in \mathbb{R}^{m \times m}$ where $m \ll N$. The major CPU cost for constructing the local model is mainly one LU decomposition for inverting $\mathbf{E}_{\mathbf{0}}$ and $d$ times forward and backward substitution.

In order to apply Monte-Carlo simulation, parameters $\tilde{\zeta}$ will be first calculated after generating the original parameters $\tilde{\xi}$, then substituting $\tilde{\zeta}$ into the reduced system (4.22). The output of interest with given parameters in the selected frequency can be evaluated by solving the reduced system

$$
\begin{aligned}
\tilde{\mathbf{X}} & =\left(\tilde{\mathbf{E}}+\zeta_{1} \tilde{\mathbf{E}}_{\mathbf{1}}+\zeta_{2} \tilde{\mathbf{E}}_{\mathbf{2}}+\ldots+\zeta_{d} \tilde{\mathbf{E}}_{\mathbf{d}}\right)^{-1} \tilde{\mathbf{B}} \mathbf{U} \\
\mathbf{V} & =\tilde{\mathbf{L}}^{T} \tilde{\mathbf{X}}
\end{aligned}
$$

where

$$
\tilde{\mathbf{L}}^{T}=\mathbf{L}^{T} \mathcal{Q}
$$

Eventually the statistical results can be obtained after Monte-Carlo simulation.

To summerize, given an optimized frequency vector $\boldsymbol{f}=\left[f_{1}, f_{2}, \ldots, f_{i}\right]$, in order to calculate the statistical simulation results at one frequency point, a reduced parameterized system is obtained using an explicit multi-dimensional moment-matching and the Monte-Carlo will be applied to this reduced system. A pseudo code for the proposed PMOR using multi-dimensional moments matching is given in Algorithm 2 . 


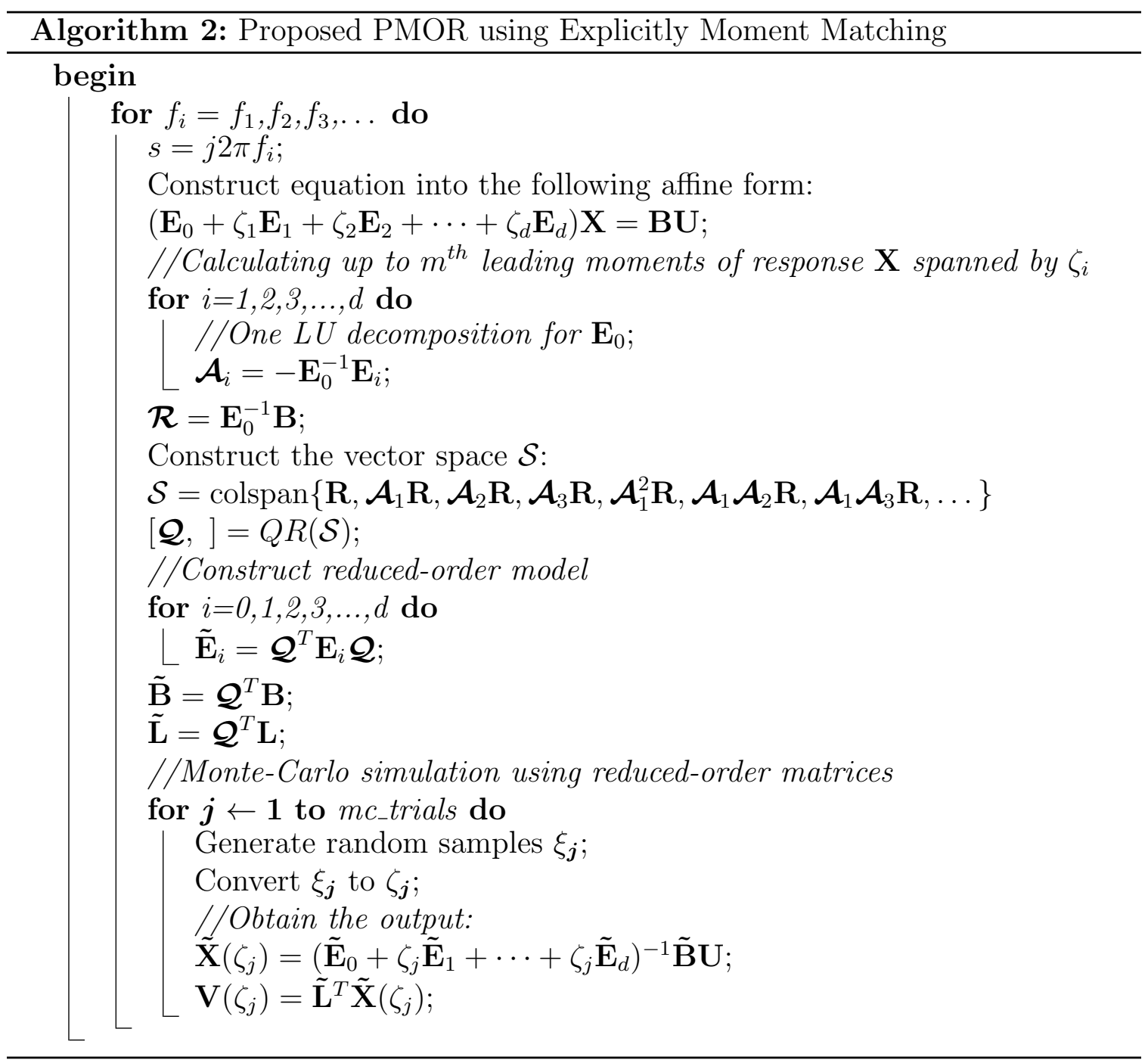




\subsubsection{Discussion}

The proposed PMOR algorithm using explicit multi-dimensional moments matching has been introduced in Section 4.3. As can be seen, the subspace $\mathcal{S}$

$$
\mathcal{S}=\operatorname{colspan}\left\{\mathbf{R}, \mathcal{A}_{1} \mathbf{R}, \mathcal{A}_{2} \mathbf{R}, \mathcal{A}_{3} \mathbf{R}, \mathcal{A}_{1}^{2} \mathbf{R}, \mathcal{A}_{1} \mathcal{A}_{2} \mathbf{R}, \mathcal{A}_{1} \mathcal{A}_{3} \mathbf{R}, \ldots\right\}
$$

is no longer a Krylov subspace and hence, the Arnoldi algorithm cannot be used to generate the orthogonal space $\mathcal{Q}$.

Another issue is that the projection operator $\mathcal{Q}$ cannot be generated without forming the subspace $\mathcal{S}$. The column vectors of the subspace $\mathcal{S}$ become easily illconditioned when the order goes high. Suppose there is only one parameter, then subspace $\mathcal{S}$ will become

$$
\mathcal{S}=\operatorname{colspan}\left\{\mathbf{R}, \mathcal{A}_{1} \mathbf{R}, \mathcal{A}_{1}^{2} \mathbf{R}, \mathcal{A}_{1}^{3} \mathbf{R}, \ldots\right\}
$$

It has been proved that the calculation of column vector $\mathcal{A}_{1}^{n} \mathbf{R}$ converges quickly to the eigenvector associated with the eigenvalue having the largest magnitude. In

the end, the moments vectors become all linearly dependent to this eigenvector $[18]$ and thus, the constructing of the moments vector space of $\mathcal{S}$ becomes impossible.

To avoid the above difficulties, the next section will introduce an implicit algorithm to compute the subspace $\mathcal{S}$. 


\subsection{Proposed Parametric Model-Order Reduction Using Implicit Moment Matching}

At a given frequency s, the output function of (4.18) can be written as

$$
\begin{aligned}
\mathbf{X} & =\left(\mathbf{I}-\zeta_{1} \mathcal{A}_{1}-\zeta_{2} \mathcal{A}_{2}-\zeta_{3} \mathcal{A}_{3}-\cdots-\zeta_{d} \mathcal{A}_{d}\right)^{-1} \mathcal{R} \mathbf{U} \\
& =\left(\mathbf{I}-\left(\sum_{i=1}^{d} \zeta_{i} \mathcal{A}_{i}\right)\right)^{-1} \mathcal{R} \mathbf{U} \\
& =\sum_{k=0}\left(\sum_{i=1}^{d} \zeta_{i} \mathcal{A}_{i}\right)^{k} \boldsymbol{R} \mathbf{U} .
\end{aligned}
$$

By defining $\mathcal{A}=\sum_{i=1}^{d} \zeta_{i} \mathcal{A}_{i}$ where $i=1,2,3, \ldots, d$, the output function becomes

$$
\mathbf{X}=\sum_{k=0} \mathcal{A}^{k} \mathcal{R} \mathbf{U}
$$

If we define $\mathbf{X}_{\rho}=\mathcal{A}^{\rho} \boldsymbol{R} \mathbf{U}$, the output function will be

$$
\mathbf{X}=\mathbf{X}_{0}+\mathbf{X}_{1}+\mathbf{X}_{2}+\ldots+\mathbf{X}_{\rho}+\ldots
$$

and $\mathbf{X}_{\rho}$ has the property that

$$
\mathbf{X}_{\rho}=\mathcal{A X} \mathbf{X}_{\rho-1}
$$

The moment vectors associated with each $\mathbf{X}_{\rho}$ will have the iterative properties shown 
as follows:

$$
\begin{aligned}
\mathcal{M}_{0} & =[\boldsymbol{R}] \\
\mathcal{M}_{1} & =\left[\mathcal{A}_{1} \mathcal{M}_{0}, \mathcal{A}_{2} \mathcal{M}_{0}, \mathcal{A}_{3} \mathcal{M}_{0}, \ldots, \mathcal{A}_{d} \mathcal{M}_{0}\right] \\
\mathcal{M}_{2} & =\left[\mathcal{A}_{1} \mathcal{M}_{1}, \mathcal{A}_{2} \mathcal{M}_{1}, \mathcal{A}_{3} \mathcal{M}_{1}, \ldots, \mathcal{A}_{d} \mathcal{M}_{1}\right] \\
\mathcal{M}_{3} & =\left[\mathcal{A}_{1} \mathcal{M}_{2}, \mathcal{A}_{2} \mathcal{M}_{2}, \mathcal{A}_{3} \mathcal{M}_{2}, \ldots, \mathcal{A}_{d} \mathcal{M}_{2}\right] \\
& \vdots \\
\mathcal{M}_{\rho} & =\left[\mathcal{A}_{1} \mathcal{M}_{\rho-1}, \mathcal{A}_{2} \mathcal{M}_{\rho-1}, \mathcal{A}_{3} \mathcal{M}_{\rho-1}, \ldots, \mathcal{A}_{d} \mathcal{M}_{\rho-1}\right]
\end{aligned}
$$

The vector space $\mathcal{S}$ from (4.21) can be written as

$$
\mathcal{S}=\operatorname{colspan}\left\{\mathcal{M}_{0}, \mathcal{M}_{1}, \mathcal{M}_{2}, \ldots, \mathcal{M}_{\rho}, \ldots\right\}
$$

Taking the advantages of this iterative property, according to [25], a Modified Gram-Schmidt algorithm can be used to generate the orthogonal projection operator $\mathcal{Q}$ of subspace $\mathcal{S}$ without explicit calculating each column moment vectors.

A pseudo code of proposed algorithm is shown in Algorithm 3 and the pseudo code of Modified Gram-Schmidt for implicit multi-dimensional moments matching [25] is given in Appendix $\mathrm{C}$. 


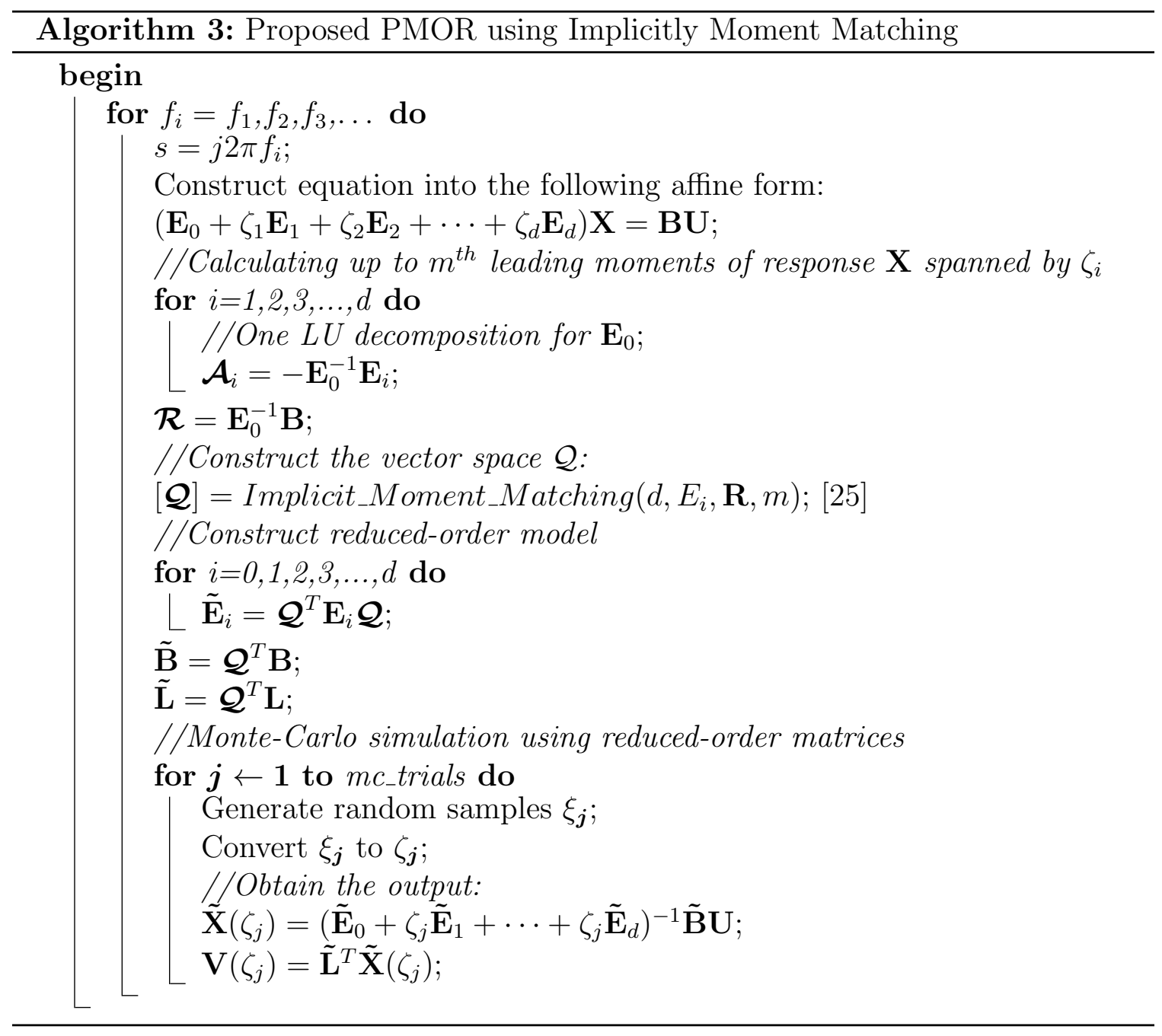




\subsection{Numerical Examples}

In this section, numerical examples are presented to show the speedup obtained by the proposed method. The CPU times reported here correspond to a PC platform with 8-GB RAM and 2-GHz Intel processor, executed in the Matlab ${ }^{\circledR}$ 8.6.0 (R2015b) environment.

\subsubsection{Example I}

In this example, we consider an interconnect structure with 4 coupled lines embedded in a circuit shown in Fig. 4.5. Each line is $8 \mathrm{~cm}$ long. The structure is modeled using 100 sections of lumped RLGC segmentation. Four $1 p F$ capacitors are connected at the far ends. The circuit is excited by an AC voltage source which is attached to the near end of the first line with $1 V$ amplitude.

Five stochastic parameters are taken into considerations. The nominal values of the stochastic physical parameters are shown in Fig. 4.5 and are assumed to have Gaussian distribution with standard deviation of $\pm 15 \%$.

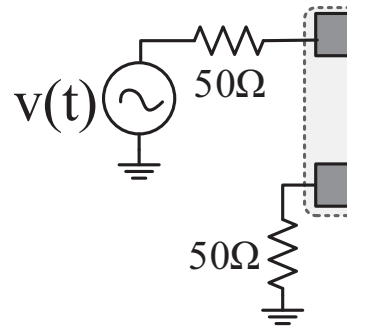

$$
\begin{aligned}
& w=0.5 \mathrm{~mm} \\
& d=2 \mathrm{~mm} \\
& h=2 \mathrm{~mm} \\
& \text { Conductivity: } \\
& \sigma=5.6 \text { E } 7 \mathrm{~S} / \mathrm{m} \\
& \text { Relative Permittivity: } \\
& \varepsilon_{r}=4.5
\end{aligned}
$$

Fig. 4.5: Four coupled interconnects circuit

The statistical distribution of the output were obtained using both the proposed and the conventional method based on 2000 Monte-Carlo simulations. The original system is reduced from $1224 \times 1224$ to a $14 \times 14$ reduced-order model using the 


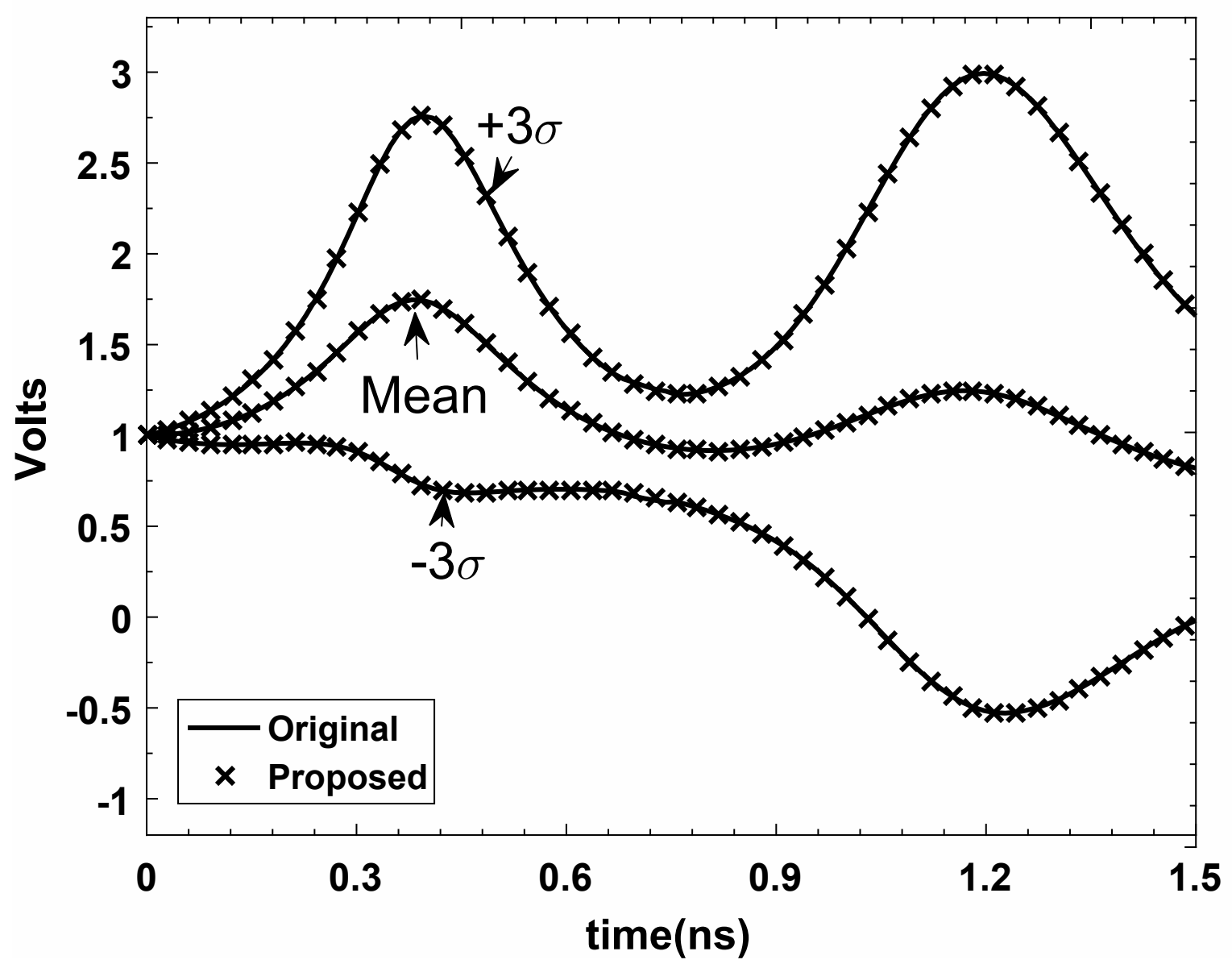

Fig. 4.6: Statistical analysis for the voltage waveform at the far-end of active line \#1 (Example 1).

proposed method. Fig. 4.6 shows the variation of the responses at the far end of both the active-line \#1 and the victim-line \#2 using the proposed and the conventional methods. As is seen, both methods are in good agreements.

Table 4.1 shows the comparison between the reduced and full models in terms of size and simulation times. As seen, the proposed method yields a speed-up of 53.8 compared to the conventional method. 
Table 4.1: CPU time and Size Comparison (Example 1)

\begin{tabular}{|l|c|c|c|c|}
\hline & Size & $\begin{array}{c}\text { Model Generation } \\
\text { Time }\end{array}$ & Simulation Time & Speed-up \\
\hline Original & 1224 & - & $592.7 \mathrm{sec}$ & - \\
\hline Proposed & 14 & $1.6 \mathrm{sec}$ & $9.4 \mathrm{sec}$ & 53.8 \\
\hline
\end{tabular}

\subsubsection{Example II}

In this example, merits of the proposed method by considering higher number of stochastic parameters is demonstrated. The same interconnect structure/model and inputs are considered while embedded in circuit shown in Fig. 4.7. The width of each transmission line as well as four other parameters $\left(d, h, \sigma, \epsilon_{r}\right)$ are taken as stochastic parameters and in total, eight stochastic parameters are considered. The parameters are assumed to have Gaussian distribution with standard deviation of $\pm \% 15$.

Fig. 4.8 shows the mean value and the $\pm 3 \sigma$ tolerances of the output responses at far-ends of both the active-line \#1 and victim-line \#2 using both the proposed method and the conventional Monte Carlo method (4000 simulations). As seen, both methods are in good agreement.

Table 4.2 shows the CPU cost of the proposed method (reduced-order model generation time + simulation time) and its comparison with the simulation time using the original model. As is seen, the proposed method yields a speed-up of 36.51 times compared to the conventional method. 


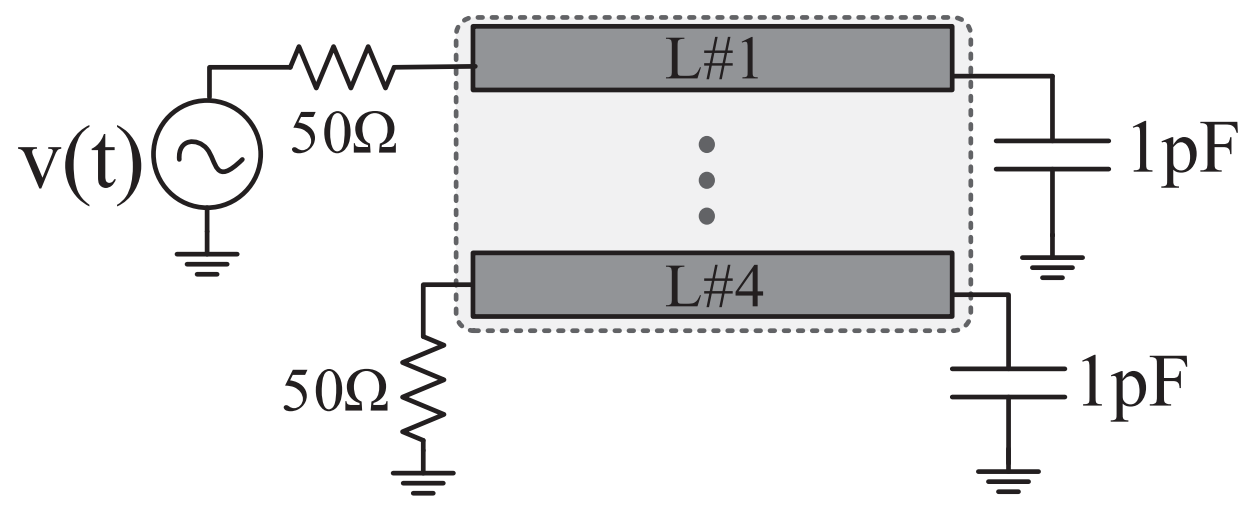

Fig. 4.7: Circuit for Example 2 (Example 2)

Table 4.2: CPU time and Size Comparison (Example 2)

\begin{tabular}{|l|c|c|c|c|}
\hline & Size & $\begin{array}{c}\text { Model Generation } \\
\text { Time }\end{array}$ & Simulation Time & Speed-up \\
\hline Original & 1224 & - & $1201.3 \mathrm{sec}$ & - \\
\hline Proposed & 18 & $2.2 \mathrm{sec}$ & $30.7 \mathrm{sec}$ & 36.51 \\
\hline
\end{tabular}




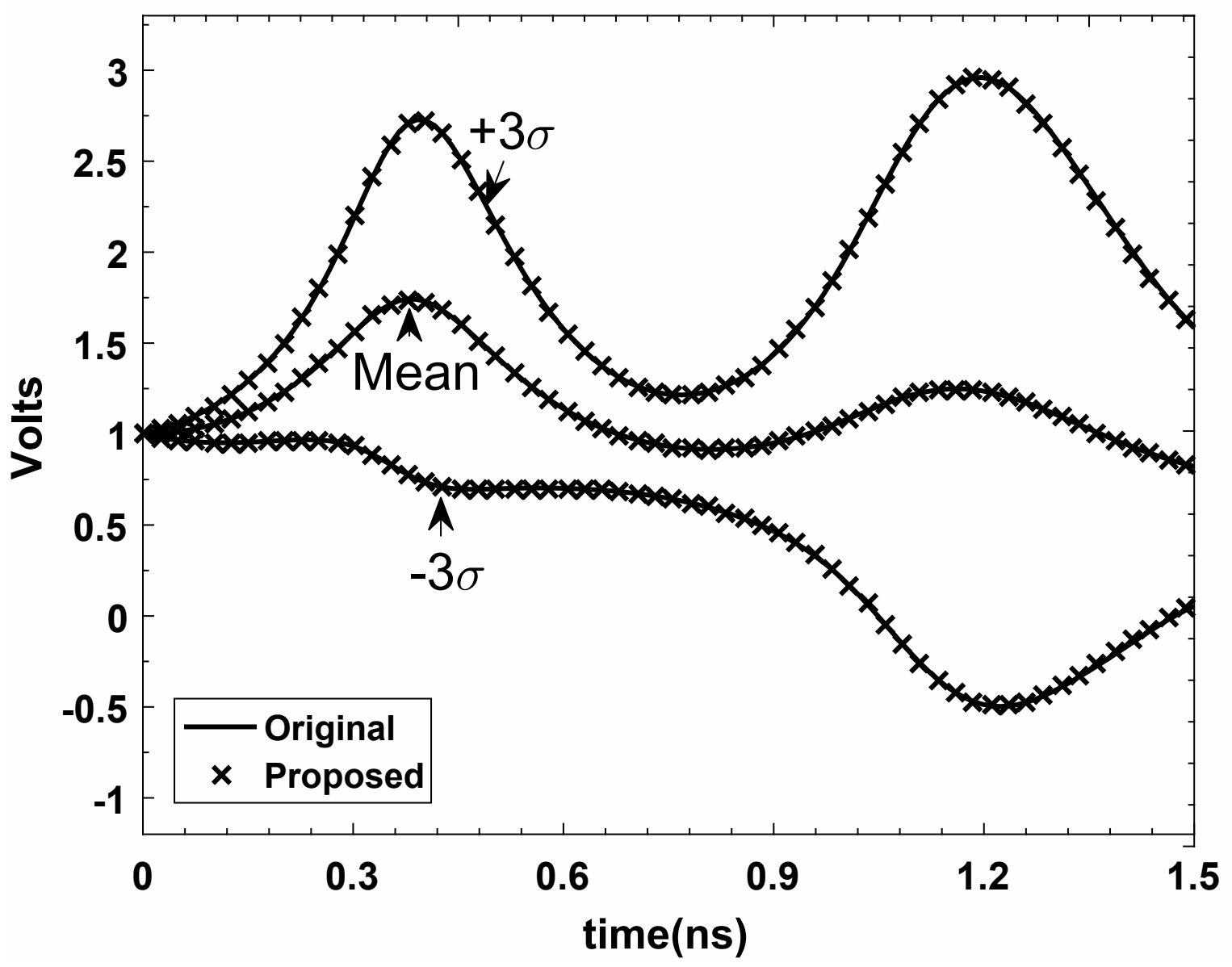

Fig. 4.8: Statistical analysis for the voltage waveform at the far-end of active line \#1 (Example 2). 


\section{Chapter 5}

\section{Proposed Time Domain Variability Analysis Using PMOR and NILT}

In Chapter 4, an efficient PMOR method was described for variability analysis in frequency domain. The proposed reduced-order model (ROM) is valid at only at the frequency under consideration. Hence the system equation at a given frequency point is an algebraic equation with respect to the parameters, and hence none of the integration methods discussed in Section 2.1.2 can be adopted (since the nature of these integration methods is to solve dynamic equations). Addressing this difficulties, in this chapter, an efficient time-domain variability analysis method combining PMOR and NILT [6] is presented.

\subsection{Concept of the Proposed Method}

One category of time-domain simulations for linear circuit is based on analyzing the circuits in frequency domain or in Laplace domain [27-32]. Since the proposed method results in a reduced model at each frequency point capturing the stochastic parameter space, one way to find the statistical information of the time-domain response is to simulate the system in frequency domain using the proposed method at different 
random parameters samples, and then use Inverse Fast Fourier Transform (IFFT) to construct the time-domain response in order to extract the required statistical information. The main drawbacks of this approach are that,

- in order to construct the time-domain response using IFFT, the frequency responses must be found for the entire band-width of interest and for each random parameter sample before applying IFFT,

- if the input to the system are waveforms with sharp edges (such as a pulse with sharp rise/fall times), a very large number of frequency points would be required in order to avoid aliasing problems [30].

Addressing the above issues, an efficient method based on Numerical-Inversion of Laplace transform (NILT) can be found in $[33,34]$. NILT method does not suffer from aliasing problems and does not require large number of frequency points to find the time-domain waveforms (typically three to five frequency points are sufficient). In addition, the method is highly parallelizable.

In this chapter, the proposed PMOR introduced in Chapter 4 is combined with the Numerical Inversion of Laplace Transform (NILT) [35] for achieving an efficient timedomain variability analysis. A brief overview of Numerical Inverse Laplace Transform is described in Section 5.2. The applications of NILT for MNA based formulation is illustrated in Section 5.3. Development of the proposed parametrized reduced-order model and its use for time-domain variability analysis are described in Sections 5.4 and 5.5. Computational results are presented in Section 5.6. 


\subsection{Numerical Inversion of Laplace Transform}

Given a Laplace domain output of a linear system $V(s)$, the time-domain response $v(t)$ may be obtained by applying the inversion of the Laplace transform on the function $V(s)$ by evaluating the following integral

$$
v(t)=\frac{1}{j 2 \pi} \int_{\alpha-j \infty}^{\alpha+j \infty} V(s) e^{s t} d s
$$

where $\alpha$ must be greater than the real part of any poles of $V(s)$, i.e., $\alpha>\Re e\left(p_{i}\right)$, where $p_{i}$ presents any poles of $V(s)$. For a stable system it is known that, all the poles of $V(s)$ exist in the left half of the complex plane. Hence, application of NILT to the passive circuits which are the focus of this thesis allows for $\alpha$ to be zero or greater, due to their intrinsic stability. By denoting a new variable $z=s t$, the inversion of laplace transform becomes,

$$
v(t)=\frac{1}{j 2 \pi t} \int_{\alpha^{\prime}-j \infty}^{\alpha^{\prime}+j \infty} V\left(\frac{z}{t}\right) e^{z} d s
$$

where $\alpha^{\prime}$ is an arbitrary constant such that $\alpha^{\prime}>\Re e\left(p_{i}\right)$. Exact inversion may only be executed once all the poles of $V(s)$ are known. However, this presents a challenge as large circuits may possess numerous poles and it proves to be prohibitively computationally expensive to apply root finding procedures to $V(s)$.

Alternately, the integral in (5.2) can be evaluated numerically using Numerical Inversion of Laplace Transform (NILT) presented in $[35,36]$ and its main idea is illustrated in Fig. 5.1.

First, approximating the exponential function $e^{z}$ using the Padé approximation 


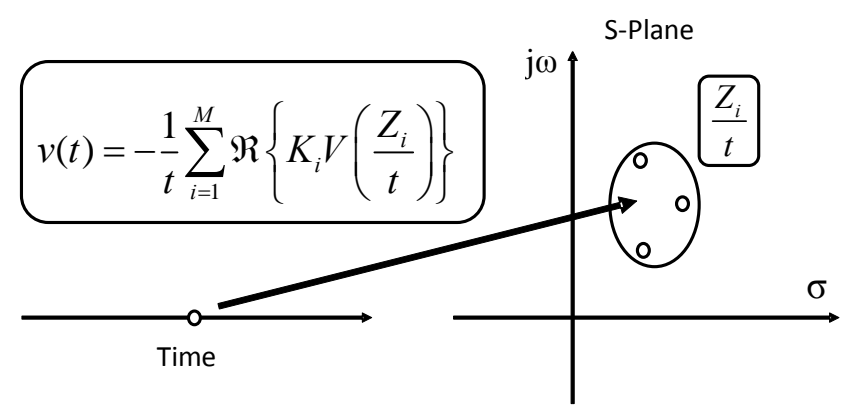

Fig. 5.1: An illustration of NILT that the time response at one time point is a summation of frequency responses at several complex frequencies.

we get

$$
\begin{aligned}
e^{z} & \approx R_{N, M}(z)=\frac{P_{N}(z)}{Q_{M}(z)}=\frac{\sum_{i=0}^{N}(M+N-i) !\left(\left(\begin{array}{c}
N \\
i
\end{array}\right)\right) z^{i}}{\sum_{i=0}^{M}(-1)^{i}(M+N-i) !\left(\left(\begin{array}{c}
M \\
i
\end{array}\right) z^{i}\right.} \\
& =\sum_{n=1}^{M} \frac{K_{i}}{z-z_{i}}
\end{aligned}
$$

where where $P_{N}(z)$ and $Q_{M}(z)$ are polynomials of order $N$ and $M$, respectively; $z_{i}$ and $k_{i}$ denote the poles and residues of rational function $\frac{P_{N}(z)}{Q_{M}(z)}$, respectively.

Substituting $e^{z} \approx R_{N, M}(z)$ into $(5.2), v(t)$ can be written as

$$
v(t)=\frac{1}{j 2 \pi t} \int_{\alpha-j \infty}^{\alpha+j \infty} \mathbf{V}(z / t) R_{N, M}(z) d z
$$

In accordance with the residue theorem the integral may be evaluated by closing the path of integration between $\alpha^{\prime}-j \infty$ and $\alpha^{\prime}+j \infty$ either to the left or to the right. By the Cauchy theorem only the residues within the closed contour will contribute to the integral. Here, the choice is made to close the path to the right as there are numerous residues in the left half plane arising from the system's poles in the left half plane. In order that the path along the infinite arc not contribute to the integral, it is necessary that $M, N$ be chosen such that the function $V(z / t) R_{N, M}(z)$ has at least 
two more finite poles than zeroes.

By evaluating the integral in (5.4) through a closed path integration around the poles of $R_{N, M}(z)$ in the right half plane, $v(t)$ can be approximated as

$$
\tilde{v}(t) \approx-\frac{1}{t} \sum_{i=1}^{W} \Re e\left\{K_{i} \mathbf{V}\left(z_{i} / t\right)\right\}
$$

where

$$
W= \begin{cases}M / 2, & \text { if } M \text { is even } \\ (M+1) / 2 & \text { if } M \text { os odd }\end{cases}
$$

NILT can be used as an A-stable high-order integration method of solving differential equations with order equivalent to $M+N$ [37]. Therefore NILT has several advantages comparing with traditional LMS method (e.g., Backward Euler or Trapezoidal rule). One advantage is that, since the truncation error corresponds to the step-size and the order of integration method, NILT can take relatively large stepsize compared to the LMS method. The other advantage is that, the response at any time point using NILT is independent of previous time points; therefore this method is highly suitable for parallel simulation. In addition, normally 3 to 5 conjugate poles are sufficient to estimate a time response. A discussion of stability and error estimation of NILT can be found in $[33,37]$ and are given in Appendix D and E, respectively. 


\subsection{Time-domain Statistical Analysis using NILT}

MNA equations in the Laplace domain can be expressed as:

$$
\begin{aligned}
(\mathbf{G}+s \mathbf{C}) X(s) & =\mathbf{B} U(s) \\
\mathbf{V}(\mathbf{s}) & =\mathbf{L}^{T} X(s),
\end{aligned}
$$

where $\mathbf{G}$ and $\mathbf{C}$ are admittance and susceptance matrices, $\mathbf{B}$ is the selector matrix and $U(s)$ is the input source in Laplace domain.

Due to physical and environmental tolerances, values of circuit components can deviate from their nominal values. This deviation is random in nature, leading to uncertainty in the circuit response. In order to characterize the statistics of uncertainty in the circuit response, the MNA equations are modified to include the random parameters as

$$
\begin{aligned}
\mathbf{E}(s, \xi) \mathbf{X}(s, \xi) & =\mathbf{B U}(s) \\
\mathbf{V}(s, \xi) & =\mathbf{L}^{T} \mathbf{X}(s, \xi)
\end{aligned}
$$

where $\mathbf{E}(s, \xi)=\mathbf{G}(\xi)+s \mathbf{C}(\xi)$ and $\xi=\left[\xi_{1}, \ldots, \xi_{d}\right]$ is a d-dimensional vector collecting all the random circuit parameters.

Solutions of equation (5.7) can be obtained as:

$$
\mathbf{V}(s, \xi)=\mathbf{L}^{T} \mathbf{E}^{-1}(s, \xi) \mathbf{B U}(s)
$$

In order to obtain the time domain response $v(t)$ at $t$ using NILT, frequency 
response is evaluated by substituting

$$
s=\frac{s_{i}}{t}
$$

into (5.9), where $s_{i}$ are poles of Padé approximation of $e^{z}$. The time response can be evaluated by substituting $\mathbf{V}\left(\frac{z_{i}}{t_{o}}\right)$ in (5.9) into (5.5) as

$$
v(t, \xi) \approx-\frac{1}{t} \sum_{i=1}^{M} K_{i} \mathbf{L}^{T} \mathbf{E}^{-1}\left(\frac{s_{i}}{t}, \xi\right) \mathbf{B U}\left(\frac{s_{i}}{t}\right) .
$$




\subsection{Orthogonal Matrix Calculation}

In this section, we show how to implicitly find the block moments of the system based on the method described by Section 4.3 which is suitable for adoption in an NILT-based analysis.

Consider the MNA equation (5.8) of the system and expressing $\mathbf{E}(s, \xi)$ at a specific frequency $s$ as:

$$
\mathbf{E}(s, \xi)=\mathbf{E}_{0}(s)+\zeta_{1} \mathbf{E}_{1}(s)+\zeta_{2} \mathbf{E}_{2}(s)+\ldots+\zeta_{d} \mathbf{E}_{d}(s)
$$

where $\zeta_{i}$ are some functions of $\xi$ and $\mathbf{E}_{i}(s)$ are constants.

Substituting (5.12) into (5.8) results in the following equation for the output function $\mathbf{V}(s, \xi)$ :

$$
\begin{aligned}
\mathbf{V}(s, \zeta)= & \mathbf{L}^{T}\left(\mathbf{E}_{\mathbf{0}}(s)+\zeta_{1} \mathbf{E}_{\mathbf{1}}(s)+\zeta_{2} \mathbf{E}_{\mathbf{2}}(s)+\ldots\right. \\
& \left.+\zeta_{d-1} \mathbf{E}_{d-1}(s)+\zeta_{d} \mathbf{E}_{d}(s)\right)^{-1} \mathbf{B} \mathbf{U} \\
= & \mathbf{L}^{T}\left(\mathbf{I}-\zeta_{1}\left(-\mathbf{E}_{0}^{-1}(s) \mathbf{E}_{1}(s)\right)\right. \\
& -\zeta_{2}\left(-\mathbf{E}_{0}^{-1}(s) \mathbf{E}_{2}(s)\right)- \\
& \left.\cdots-\zeta_{d}\left(-\mathbf{E}_{0}^{-1}(s) \mathbf{E}_{d}(s)\right)\right)^{-1} \mathbf{E}_{0}^{-1}(s) \mathbf{B U}
\end{aligned}
$$

By defining $\mathcal{A}_{i}(s)=-\mathbf{E}_{0}^{-1}(s) \mathbf{E}_{i}(s), i=1,2,3, \ldots, d$ and $\mathcal{R}_{0}(s)=\mathbf{E}_{0}^{-1}(s) \mathbf{B},(5.13)$ can be written as 


$$
\begin{gathered}
\mathbf{V}(s, \zeta)=\mathbf{L}^{T}\left(\mathbf{I}-\zeta_{1} \mathcal{A}_{1}(s)-\zeta_{2} \mathcal{A}_{2}(s)-\right. \\
\left.\cdots-\zeta_{d} \mathcal{A}_{d}(s)\right)^{-1} \mathcal{R}_{0}(s) \mathbf{U} \\
=\mathbf{L}^{T}\left(\mathbf{I}-\left(\sum_{\eta=1}^{d} \zeta_{\eta} \mathcal{A}_{\eta}(s)\right)\right)^{-1} \mathcal{R}_{0}(s) \mathbf{U}
\end{gathered}
$$

Re-writing (5.14) as a power series yields:

$$
\begin{aligned}
\mathbf{V}(s, \zeta) & =\mathbf{L}^{T} \sum_{\rho=0}^{\infty}\left(\sum_{\eta=1}^{d} \zeta_{\eta} \mathcal{A}_{\eta}(s)\right)^{\rho} \mathcal{R}_{0}(s) \mathbf{U} \\
& =\mathbf{L}^{T}\left(\mathcal{R}_{0}(s)+\sum_{\rho=1}^{\infty}\left(\sum_{\eta=1} \zeta_{\eta} \mathcal{A}_{\eta}(s)\right)^{\rho} \mathcal{R}_{0}(s)\right) \mathbf{U}
\end{aligned}
$$

Using (5.15), the subspace spanned by the columns of the coefficient of the parameters can be defined as

$$
\gamma(s)=\operatorname{colspan}\left\{\mathcal{R}_{0}(s), \mathcal{R}_{1}(s), \cdots, \mathcal{R}_{\rho}(s), \cdots\right\}
$$

where

$$
\begin{aligned}
& \mathcal{R}_{\rho}(s)= \\
& {\left[\mathcal{A}_{1}(s) \mathcal{R}_{\rho-1}(s), \mathcal{A}_{2}(s) \mathcal{R}_{\rho-1}(s), \cdots, \mathcal{A}_{d}(s) \mathcal{R}_{\rho-1}(s)\right]}
\end{aligned}
$$

The form of the subspace $\gamma(s)$ allows an implicit-moment matching by using an implicit recursive modified Gram-Schmidt-like process [4] to construct a projection matrix $\mathbf{Q}_{i}(s)$ at the expansion points, associated with a frequency sample $s$. The reduced-order model can then be found as follows 


$$
\hat{\mathbf{X}}(s, \zeta)=\left(\hat{\mathcal{R}}_{0}(s)+\sum_{\rho=1}^{\infty}\left(\sum_{\eta=1} \zeta_{\eta} \hat{\mathcal{A}}_{\eta}(s)\right)^{\rho} \hat{\mathcal{R}}_{0}(s)\right) \mathbf{U}(s)
$$

where

$$
\begin{aligned}
& \hat{\mathcal{R}}_{0}(s)=\mathbf{Q}_{i}^{T}(s) \mathcal{R}_{0}(s) \\
& \hat{\mathcal{A}}_{\eta}(s)=\mathbf{Q}_{i}^{T}(s) \mathcal{A}_{\eta}(s) \mathbf{Q}_{i}(s)
\end{aligned}
$$

Then the output of interest can be computed using

$$
\mathbf{V}(s, \zeta)=\hat{\mathbf{L}}^{T}(s) \hat{\mathbf{X}}(s, \zeta)
$$

where $\hat{\mathbf{L}}^{T}(s)=\mathbf{L}^{T} \mathbf{Q}(s)$.

The approximated response in (5.18) at the given frequency point $s$ matches the leading parameter-moments of the original response in (4.20). Since the stochastic parameters appear as symbolic variables in (5.18), the statistical analysis of the circuit can be efficiently obtained using the reduced-order models in (5.18), instead of the full model in (4.20). 


\subsection{Time-domain Variability Analysis Using PMOR and NILT}

Consider the MNA equation in (5.7) written in the time-domain as follows.

$$
\begin{aligned}
\mathbf{C}(\xi) \frac{d}{d t} \mathbf{x}(t, \xi)+\mathbf{G}(\xi) \mathbf{x}(t, \xi) & =\mathbf{B u}(t) \\
v(t, \mathbf{P}) & =\mathbf{L}^{T} \mathbf{x}(t, \xi)
\end{aligned}
$$

For a given time point $t$, the circuit response $\mathbf{v}(t)$ can be evaluated as a linear combination of the responses at $M$ complex frequencies $s=z_{i} / t$ for $i=1, \ldots, M$ as

$$
\mathbf{v}(t, \xi) \approx-\frac{1}{t} \sum_{i=1}^{M} \Re e\left\{K_{i} \mathbf{V}(s, \xi)\right\}
$$

where $\mathbf{V}(s, \xi)$ is the response of the parameterized circuit in (5.12) at complex frequency $s \in\left\{s_{1}, \ldots, s_{M}\right\}$, and is obtained using (5.7) as

$$
\begin{aligned}
\mathbf{E}(s, \xi) \mathbf{X}(s, \xi) & =\mathbf{B} \mathbf{U}(s) \\
\mathbf{V}(s, \xi) & =\mathbf{L}^{T} \mathbf{X}(s, \xi)
\end{aligned}
$$

Using the proposed parametric model order reduction scheme, projection operator $\mathbf{Q}(s)$ will be constructed at each complex frequency $s \in\left\{s_{1}, \ldots, s_{M}\right\}$. The original $N$-dimensional system defined in (5.23) is projected on a reduced subspace through the approximation $\hat{\mathbf{X}}(s, \xi) \approx \mathbf{Q}^{T}(s) \mathbf{X}(s, \xi)$.

The resulting parameterized reduced-order models at complex frequency point $s$ 
for $i=1, \ldots, M$ are in the form

$$
\begin{aligned}
\hat{\mathbf{E}}(s, \xi) \hat{\mathbf{X}}(s, \xi) & =\hat{\mathbf{B}} \mathbf{U}(s) \\
\mathbf{V}(s, \xi) & \approx \hat{\mathbf{L}}^{T} \hat{\mathbf{X}}(s, \xi)
\end{aligned}
$$

where

$$
\hat{\mathbf{B}}(s)=\mathbf{Q}(s)^{T} \mathbf{B}, \quad \hat{\mathbf{L}}^{T}(s)=\mathbf{L}^{T} \mathbf{Q}(s)
$$

and

$$
\hat{\mathbf{E}}(s, \xi)=\mathbf{Q}(s)^{T} \mathbf{E}(s, \xi) \mathbf{Q}(s)
$$

Hence, the approximate response from the reduced-order model (5.24) is given by

$$
\mathbf{V}(s, \xi) \approx \hat{\mathbf{L}}^{T}(s)(\hat{\mathbf{E}}(s, \xi))^{-1} \hat{\mathbf{B}} \mathbf{U}(s)
$$

After evaluating the frequency response $\mathbf{V}(s, \xi)$ at each frequency point, the timedomain response $\mathbf{v}(t, \xi)$ can be obtained using

$$
\mathbf{v}(t, \xi) \approx-\frac{1}{t} \sum_{i=1}^{M} \Re e\left\{K_{i} \mathbf{V}(s, \xi)\right\}
$$

It is to be noted that the response at any given time point is calculated from $M$ independent evaluations of $V(s)$. In addition, the computation of the response at two separate time points can be done completely independently as two separate processes, providing a straightforward coarse parallel processing ability.

A summary of the steps associated with the proposed algorithm is given in Algorithm 4 . 


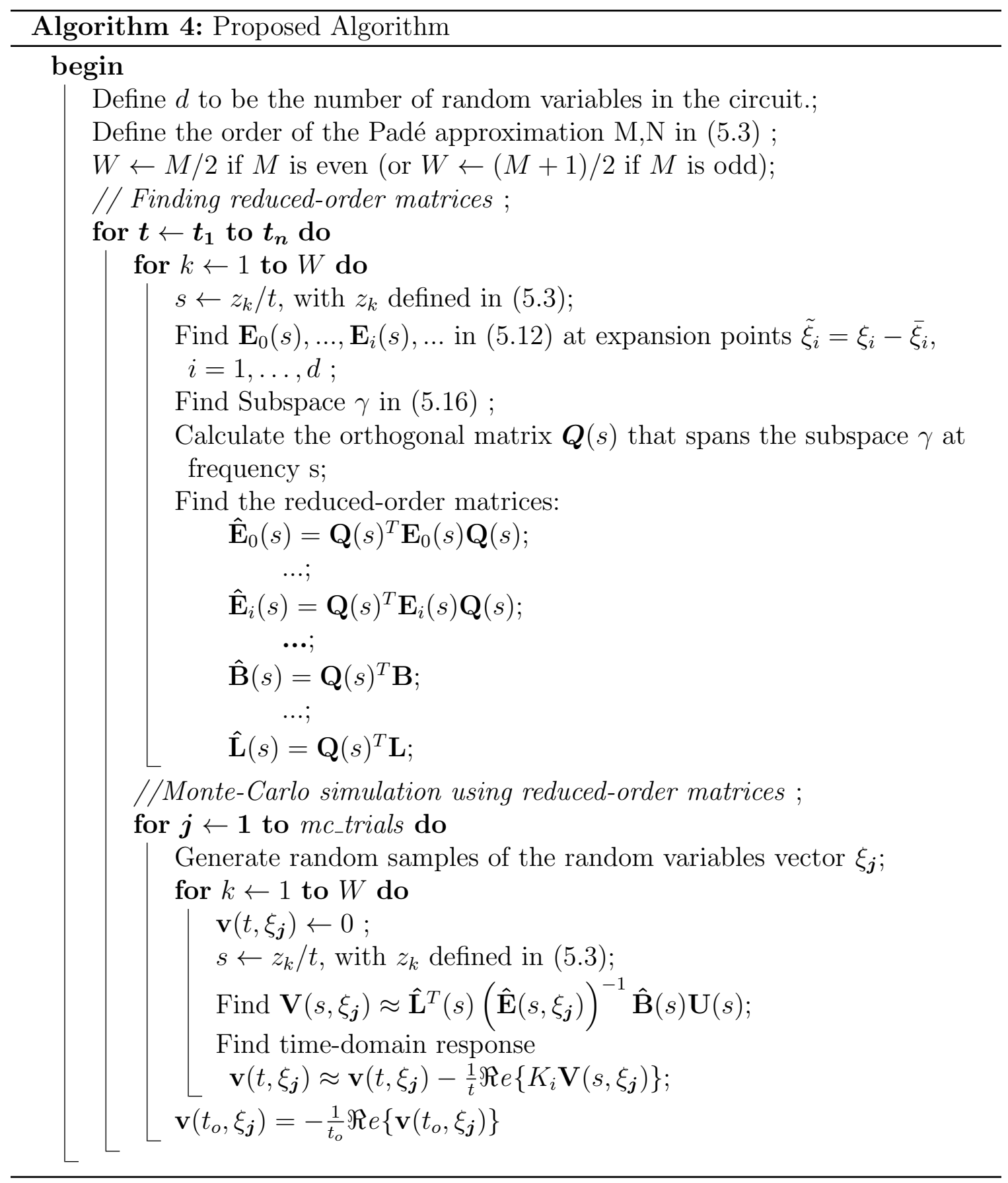




\subsection{Numerical Examples}

In this section, numerical examples are presented to show the speedup obtained by the proposed method. The CPU times reported here correspond to a PC platform

with 8-GB RAM and 2-GHz Intel processor, executed in the Matlab ${ }^{\circledR}$ 8.6.0 (R2015b) environment.

\subsubsection{Example I}

In this example, we consider an interconnect structure with 4 coupled lines embedded in a circuit shown in Fig. 5.2-(a). Each line is $8 \mathrm{~cm}$ long and modeled using 100 sections of lumped RLGC segmentation. Four $50 \Omega$ resistors are connected at the near and far ends of the lines and four $1 p F$ capacitors are connected at the far ends. The circuit is excited by a voltage source which is attached to the near end of the first line with 0.1 ns of rising/falling edge, 3ns duration and $1 V$ amplitude (shown in Fig. 5.2-(b)).

Five stochastic parameters are taken into considerations. The nominal values of the stochastic physical parameters are shown in Fig. 5.2-(a) and are assumed to have Gaussian distribution with standard deviation of $\pm 15 \%$.

The statistical distribution of the output were obtained using both the proposed and the conventional method based on 2000 Monte-Carlo simulations. Fig. 5.3 and Fig. 5.4 show the variation of the responses at the far end of both the active-line \#1 and the victim-line \#2. The original system is reduced from $1224 \times 1224$ to a $8 \times 8$ reduced-order model, while approximating the response with good accuracy.

Table 5.1 shows the comparison between the reduced and full models in terms of size and simulation times. As seen, the proposed method yields a speed-up of 62 

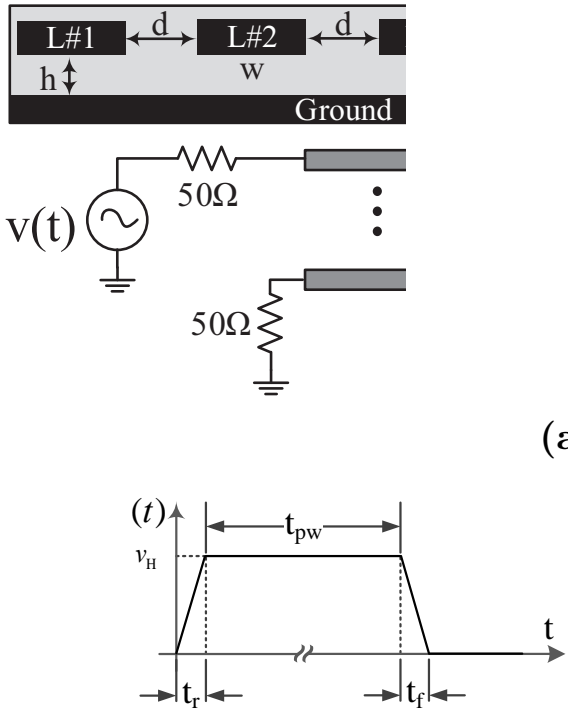

$$
\begin{aligned}
& w=0.5 \mathrm{~mm} \\
& d=2 \mathrm{~mm} \\
& h=2 \mathrm{~mm} \\
& \text { Conductivity: } \\
& \sigma=5.6 \mathrm{E} 7 \mathrm{~S} / \mathrm{m} \\
& \text { Relative Permittivity: } \\
& \varepsilon_{r}=4.5
\end{aligned}
$$

(a)

(b)

$$
\begin{aligned}
& t_{r}=t_{f}=0.1 n s \\
& t_{p w}=3 n s \\
& V_{H}=1 \mathrm{~V}
\end{aligned}
$$

Fig. 5.2: (a) Four coupled interconnects circuit, (b) Excitation waveform at input (Example 1).

Table 5.1: CPU time and Size Comparison (Example 1)

\begin{tabular}{|l|c|c|c|c|}
\hline & Size & $\begin{array}{c}\text { Model Generation } \\
\text { Time }\end{array}$ & Simulation Time & Speed-up \\
\hline Original & 1224 & - & $1934.7 \mathrm{sec}$ & - \\
\hline Proposed & 8 & $3.4 \mathrm{sec}$ & $27.9 \mathrm{sec}$ & 61.8 \\
\hline
\end{tabular}

compared to the conventional method. 


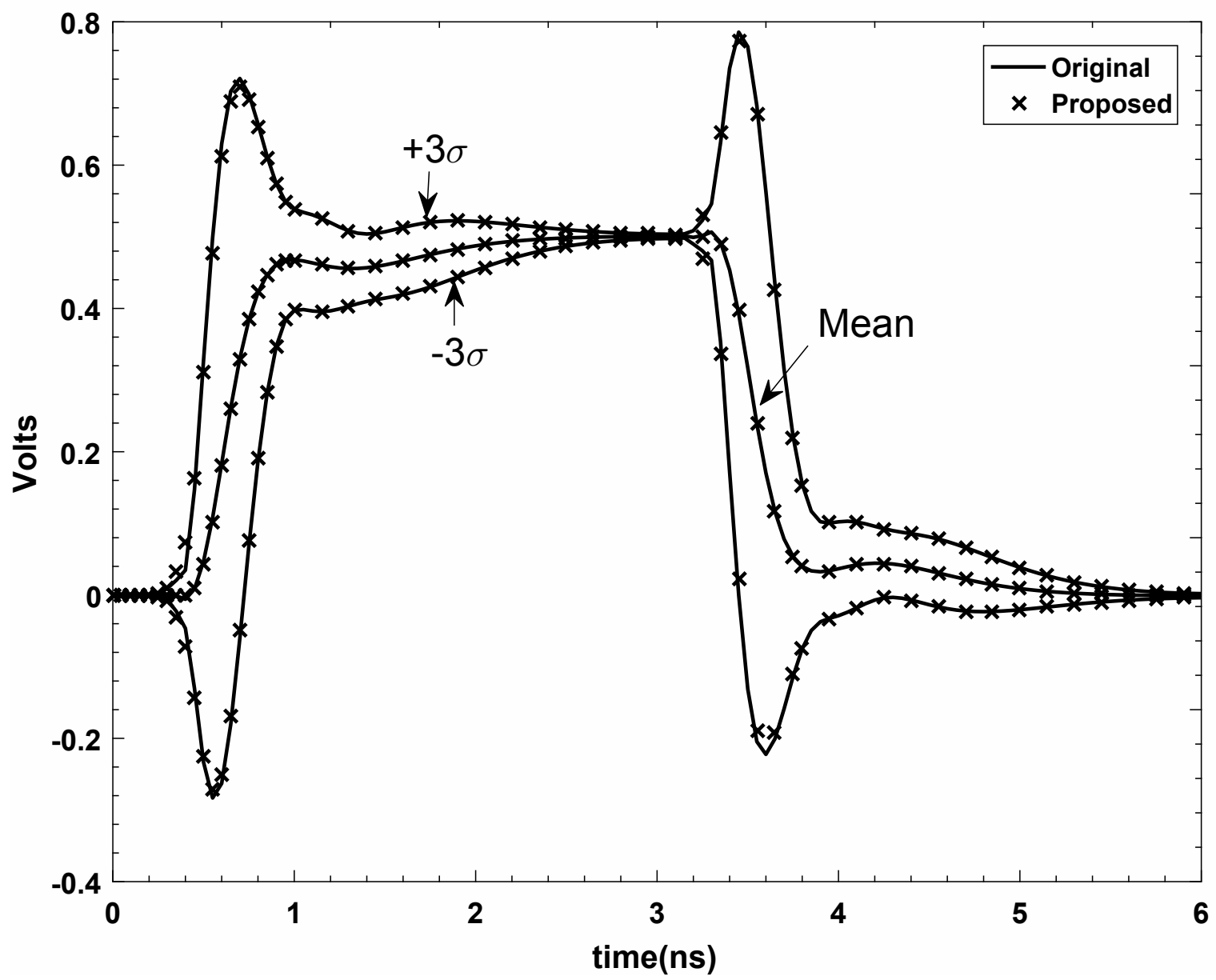

Fig. 5.3: Statistical analysis for the voltage waveform at the far-end of active line \#1 (Example 1). 


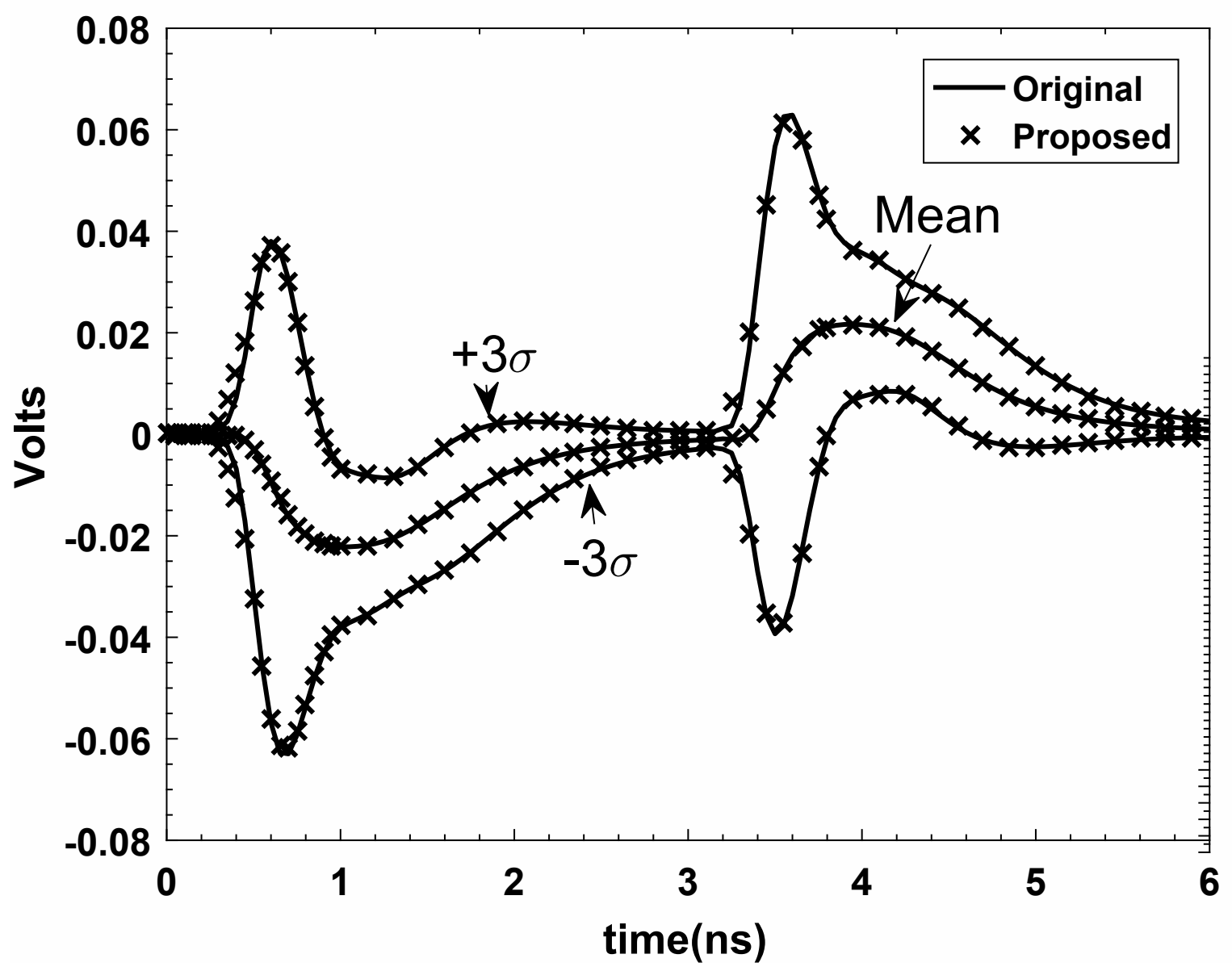

Fig. 5.4: Statistical analysis for the voltage waveform at the far-end of victim line \#2 (Example 1). 


\subsubsection{Example II}

In this example, the same interconnect structure model and inputs are considered while embedded in circuit shown in Fig. 5.5. In this case, to demonstrate the merits of the proposed method while considering higher number of stochastic parameters, the width of each transmission line is taken as a stochastic parameter and thus, eight stochastic parameters are considered. The parameters are assumed to have Gaussian distribution with standard deviation of $\pm \% 15$.

Fig. 5.6 and Fig. 5.7 shows the mean value and the $\pm 3 \sigma$ tolerances of the output responses at far-ends of both the active-line \#1 and victim-line \#2 using both the proposed method and the conventional Monte Carlo method (4000 simulations). As seen, both methods are in good agreement.

Table 5.2 shows the CPU cost of the proposed method (reduced-order model generation time and simulation time) and its comparison with the simulation time using the original model. As is seen, the proposed method yields a speed-up of 55 times compared to the conventional method.

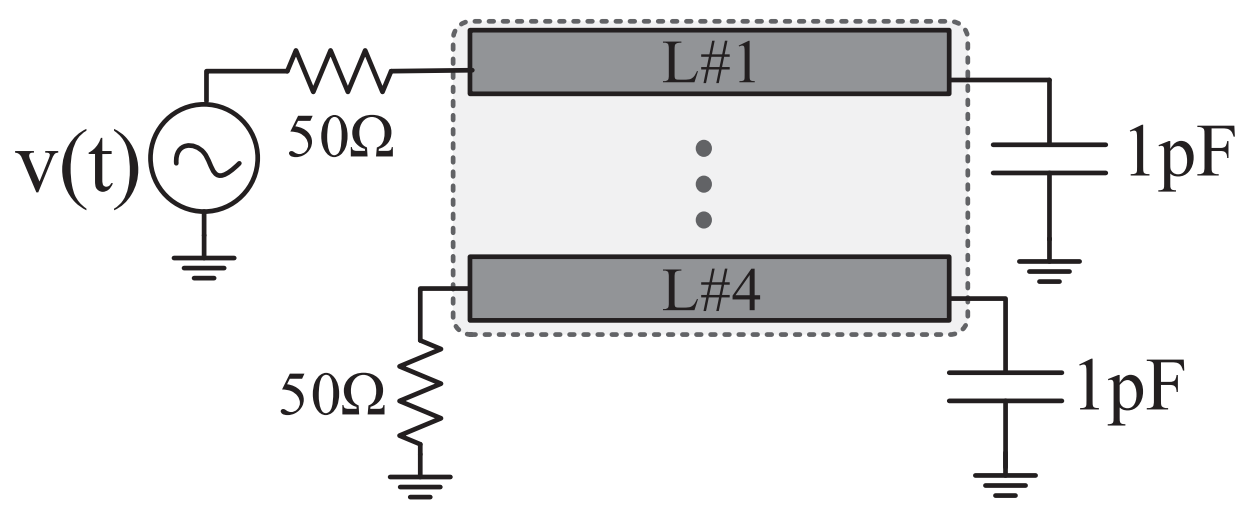

Fig. 5.5: Four coupled interconnects circuit (Example 2) 


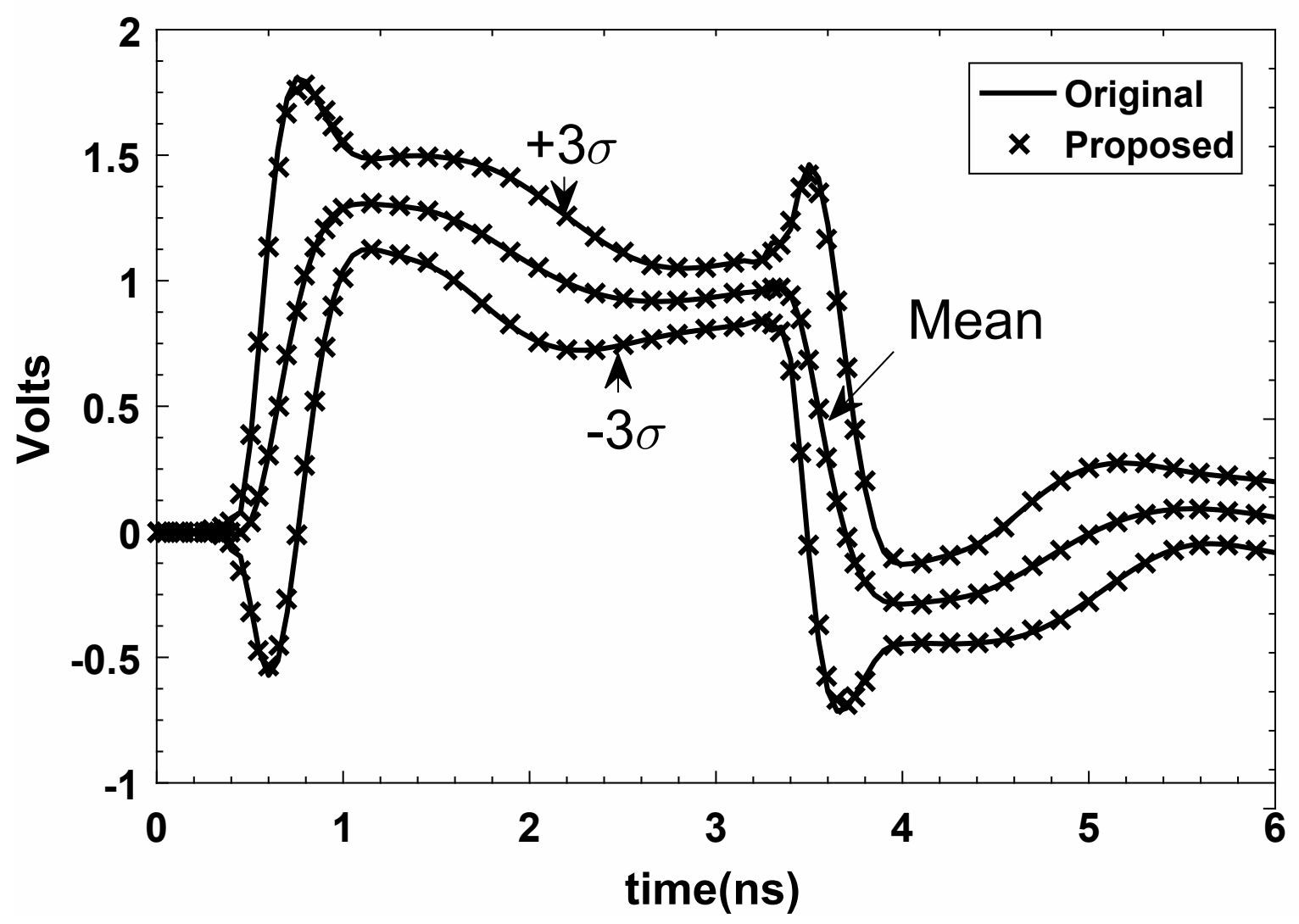

Fig. 5.6: Statistical analysis for the voltage waveform at the far-end of active line \#1 (Example 2). 


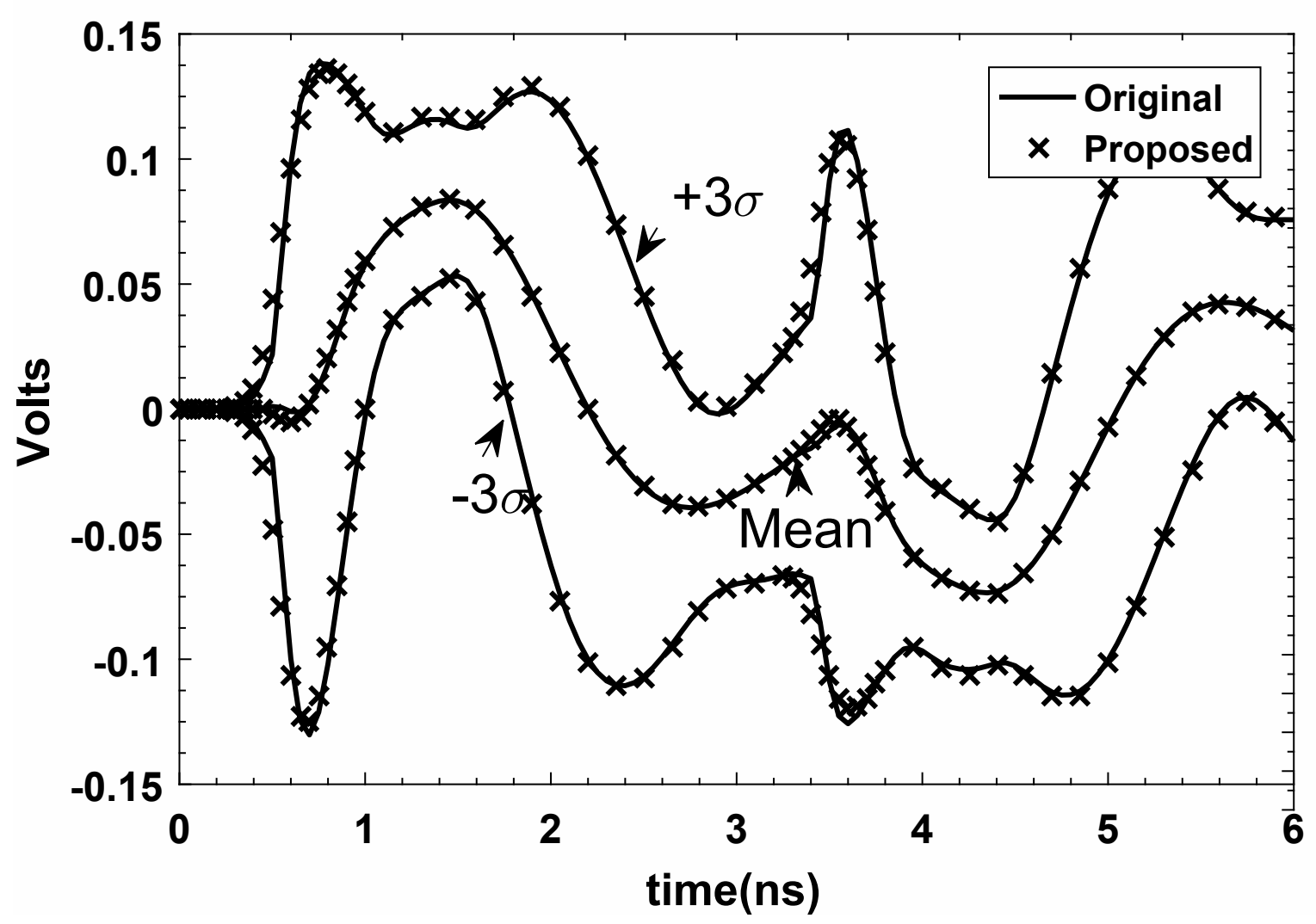

Fig. 5.7: Statistical analysis for the voltage waveform at the far-end of victim line \#2 (Example 2). 
Table 5.2: CPU time and Size Comparison (Example 2)

\begin{tabular}{|l|c|c|c|c|}
\hline & Size & $\begin{array}{c}\text { Model Generation } \\
\text { Time }\end{array}$ & Simulation Time & Speed-up \\
\hline Original & 1224 & - & $3894.4 \mathrm{sec}$ & - \\
\hline Proposed & 12 & $3.8 \mathrm{sec}$ & $67.2 \mathrm{sec}$ & 54.8 \\
\hline
\end{tabular}

\subsubsection{Example III}

In this example, the proposed method is implemented to characterize the variability of the eight transmission lines circuit shown in Fig. 5.8. The transmission lines are $10 \mathrm{~cm}$ long and modeled using 100 sections of RLGC segments. A voltage source is connected at the first of every two lines and provides a trapezoidal pusle with $0.1 n s$ rising/falling edges, $3 n s$ duration and amplitude of $1 \mathrm{~V}$. $50 \Omega$ resistors are connected at both near ends and far ends of the transmission lines and $1 p F$ capacitors are connected at the far ends. The chosen stochastic parameters are the same as in Example I and are assumed to have Gaussian distribution with standard deviation of $\pm 15 \%$.

Fig. 5.9 to Fig. 5.12 show the mean and the $\pm 3 \sigma$ tolerances of the output responses at line \#1, line \#2, line \#5 and line \#6 using both the proposed and the conventional method based on 2000 Monte Carlo simulation. As can be seen, both figures demonstrate good agreement between both methods.

Table 5.3 shows the CPU cost comparison between the proposed method and conventional method in terms of size and simulation time. The proposed method 

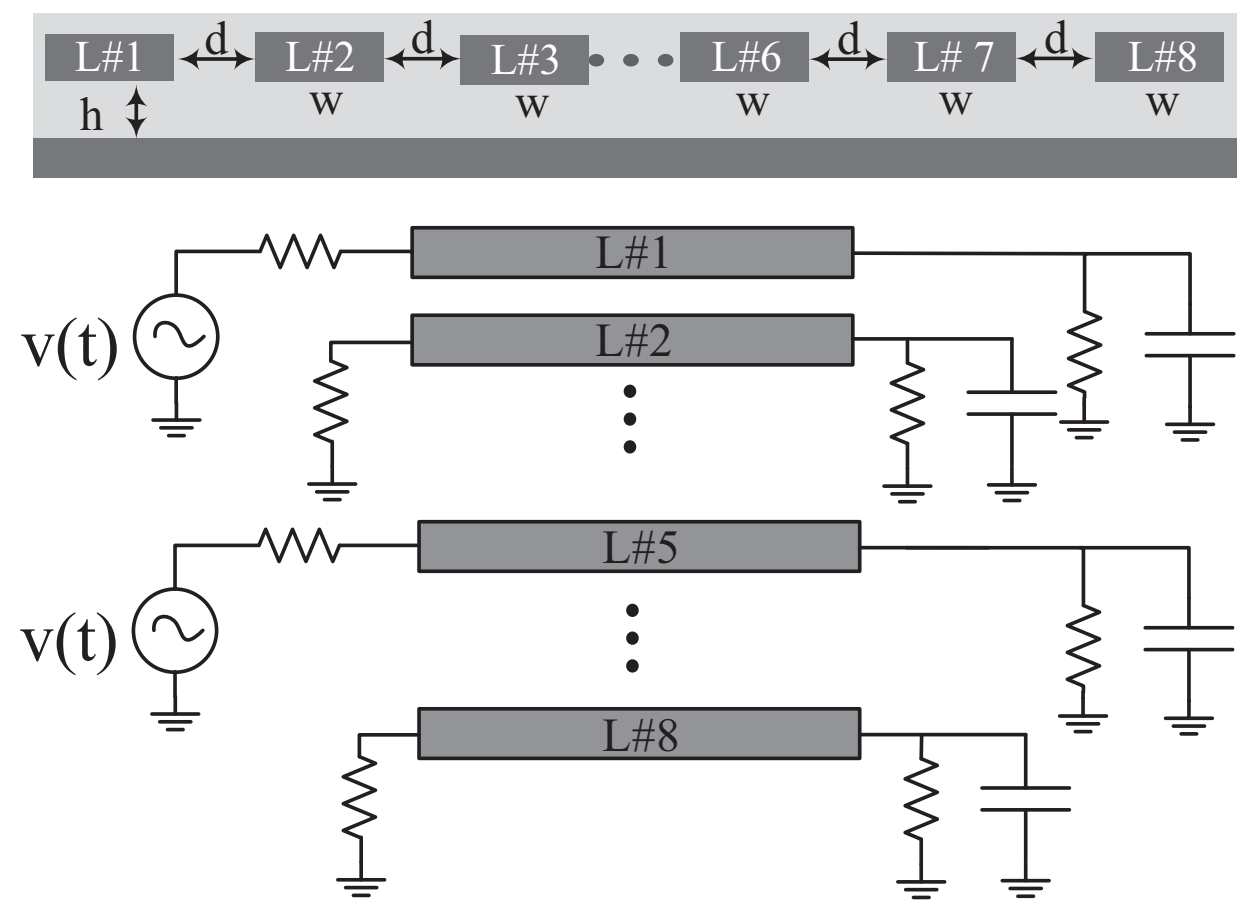

Fig. 5.8: Eight coupled interconnects circuit (Example 3).

yields a speed-up of 58 times compared to the conventional method.

Table 5.3: CPU time and Size Comparison (Example 3)

\begin{tabular}{|l|c|c|c|c|}
\hline & Size & $\begin{array}{c}\text { Model Generation } \\
\text { Time }\end{array}$ & Simulation Time & Speed-up \\
\hline Original & 2416 & - & $5817.3 \mathrm{sec}$ & - \\
\hline Proposed & 12 & $34.4 \mathrm{sec}$ & $65.2 \mathrm{sec}$ & 58.4 \\
\hline
\end{tabular}




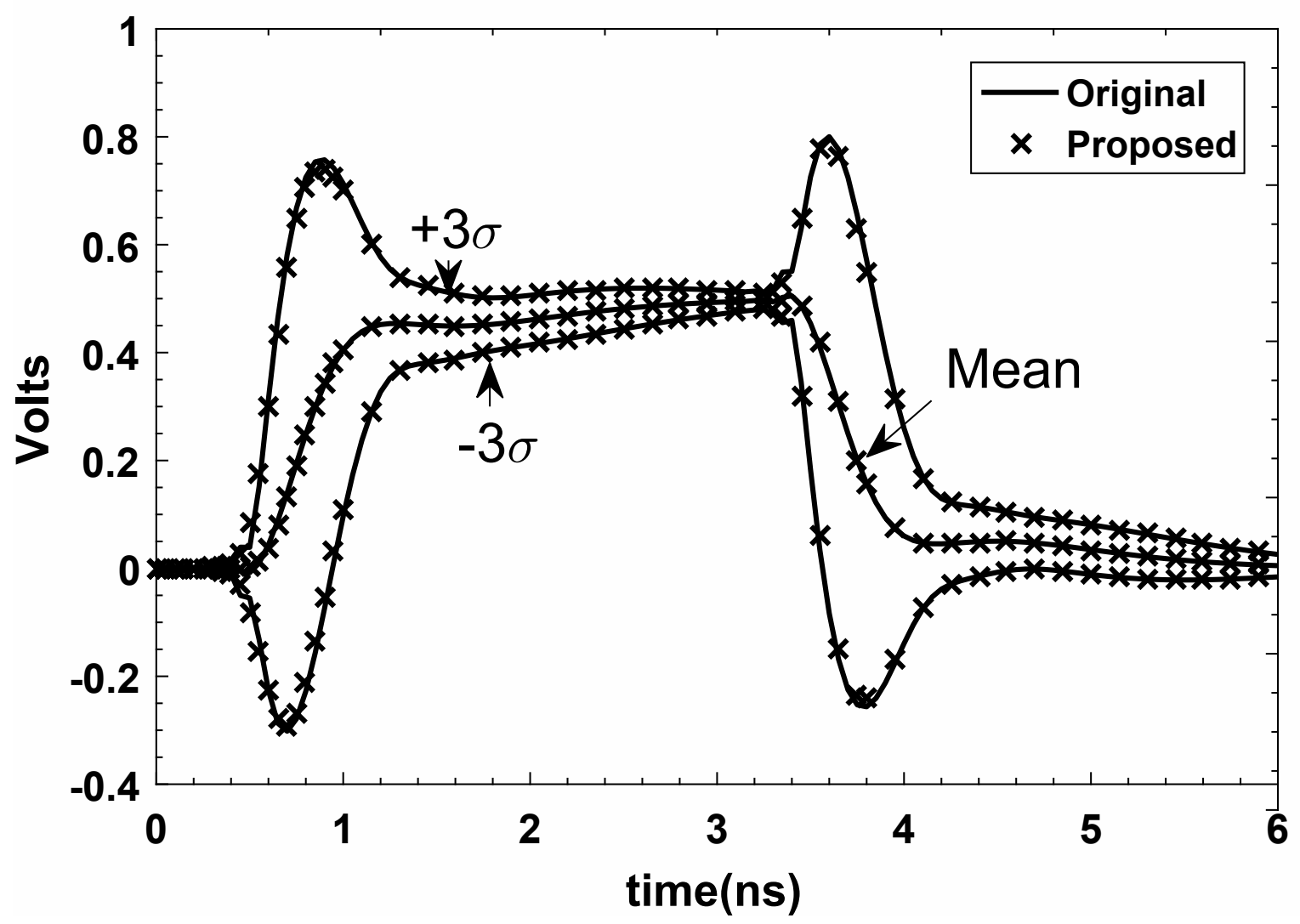

Fig. 5.9: Statistical analysis for the voltage waveform at the far-end of active line \#1 (Example 3). 


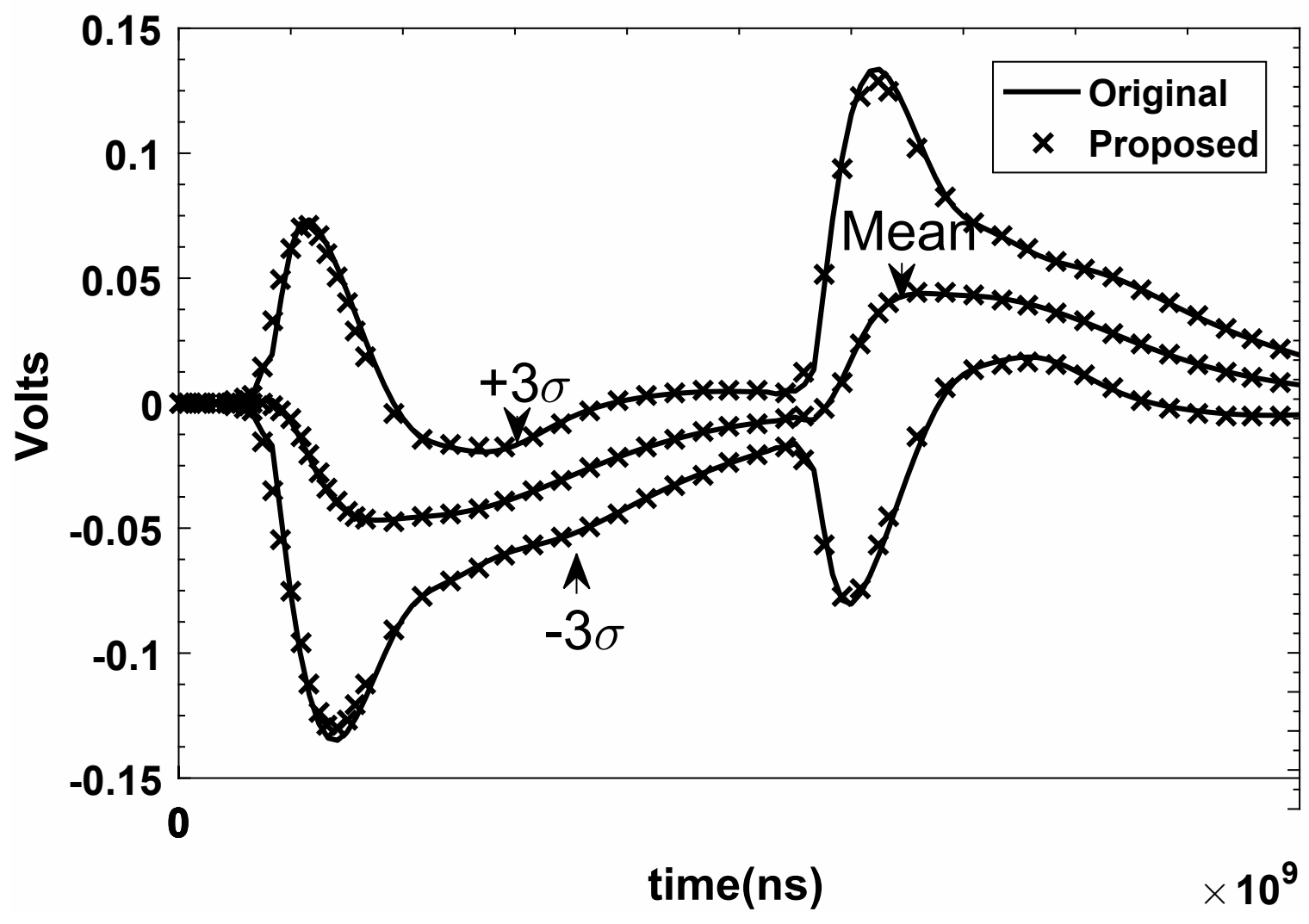

Fig. 5.10: Statistical analysis for the voltage waveform at the far-end of victim line \#2 (Example 3). 


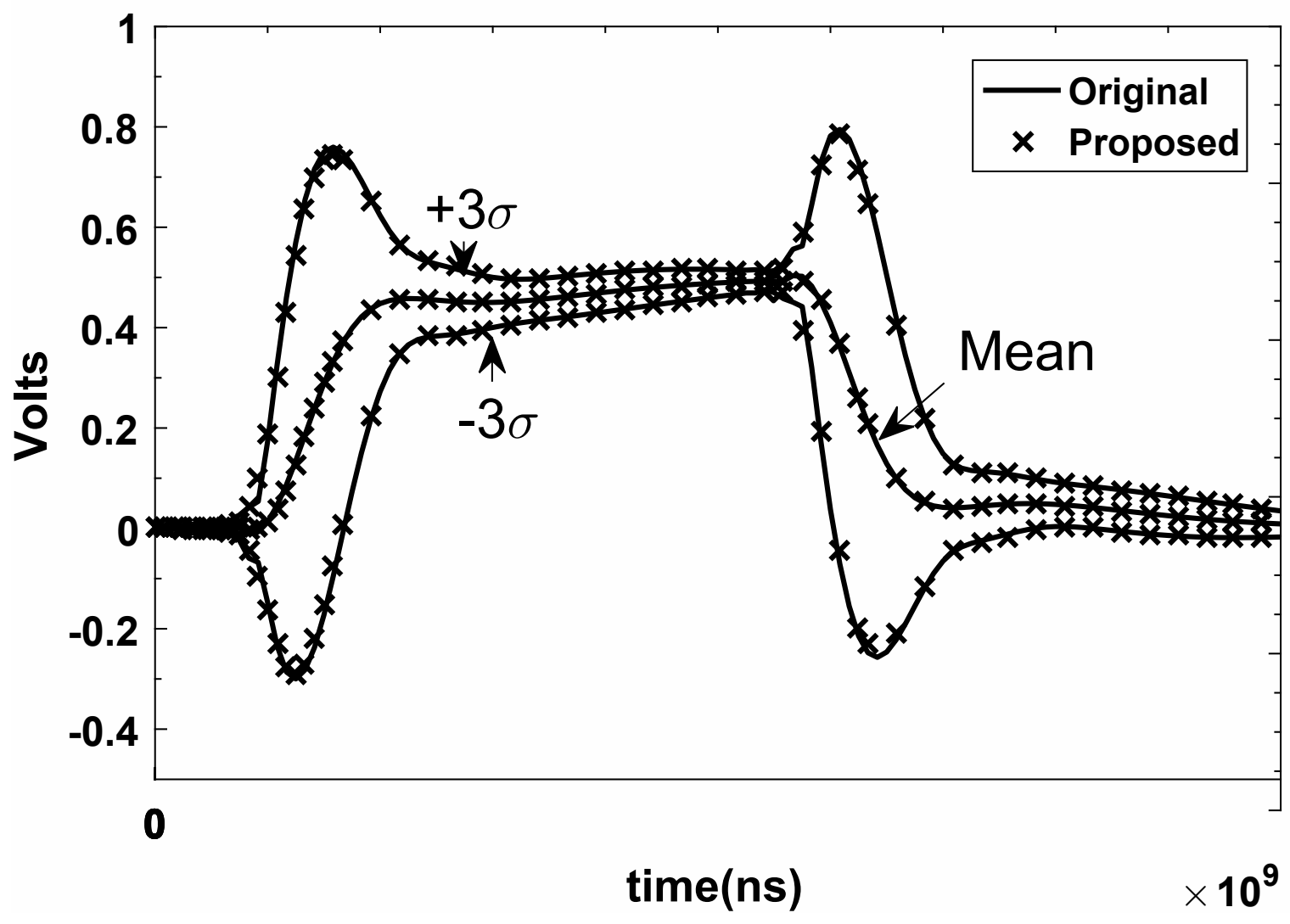

Fig. 5.11: Statistical analysis for the voltage waveform at the far-end of active line \#5 (Example 3). 


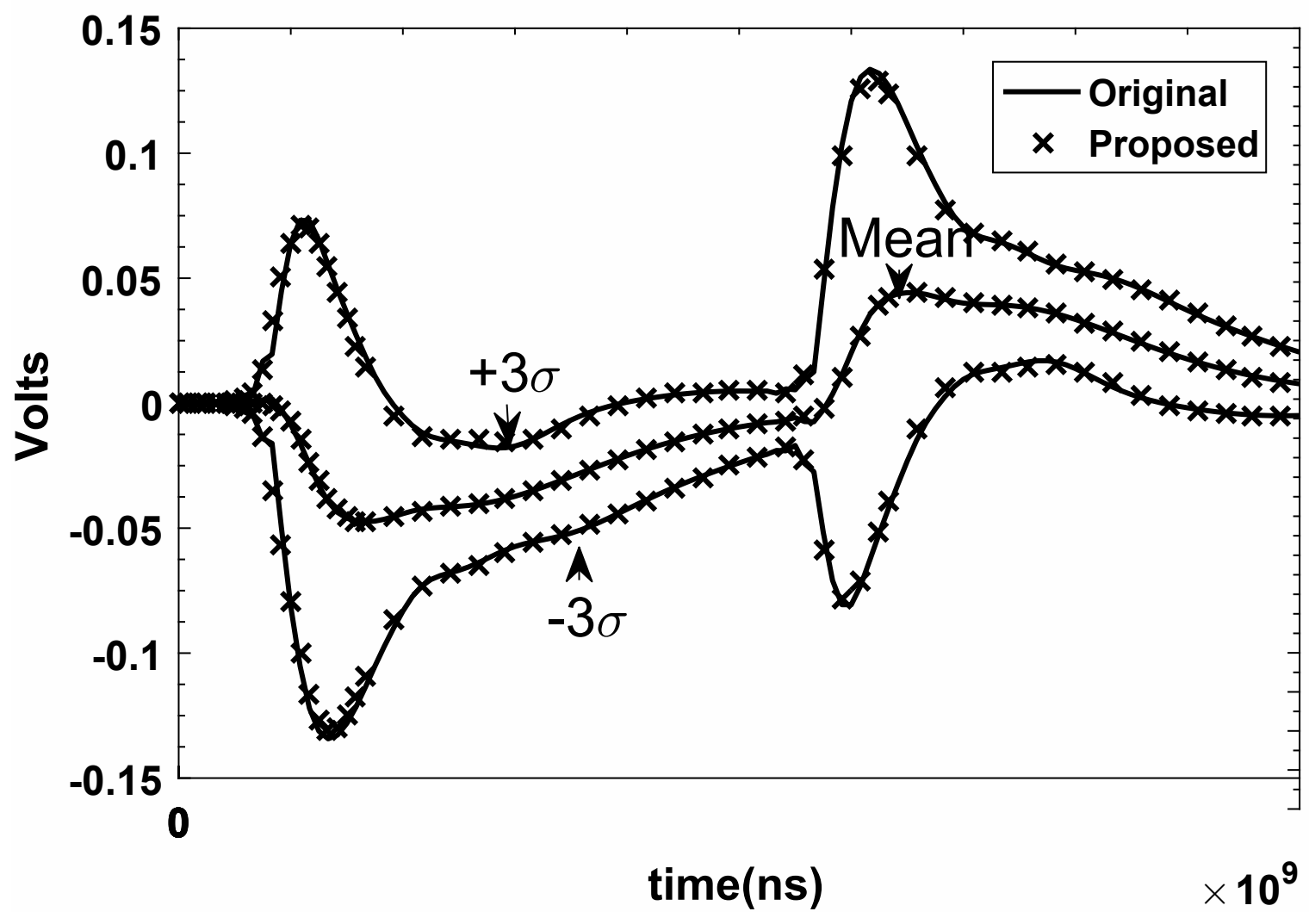

Fig. 5.12: Statistical analysis for the voltage waveform at the far-end of victim line \#6 (Example 3). 


\section{Chapter 6}

\section{Conclusions and Future Work}

\subsection{Conclusions}

One of the major constraints of variability analysis while using conventional PMOR is that the resulting reduced-order model (ROM) is dense and relatively large, thus denying higher speed-ups compared to using the original system. This thesis presented a new parametrized model order reduction algorithm for statistical analysis of linear circuits addressing the above difficulties. The key idea of the proposed PMOR is that the system response is typically a smooth function with respect to circuit stochastic parameters variations. Thus, a few number of Taylor series moments would be sufficient to accurately match the response at each frequency point. In this case, using parametrized model order reduction would result in very small reduced order models that can be used to speed-up Monte-Carlo analysis in frequency domain.

In addition, it is shown that the statistical analysis in the time domain can be easily obtained using Numerical Inversion of Laplace Transform (NILT). Since the reduced-order model obtained by the proposed PMOR only preserves the frequency at the expansion point, the reduced-order equations become algebraic equations. Addressing this issue, a new algorithm is proposed in which the PMOR is combined with 
NILT for time-domain variability analysis.

Numerical examples were presented to validate the proposed methods in both frequency domain and time domain, which shows a significantly speed-up and accuracy compared to the results obtained by original system with Monte-Carlo simulations. 


\subsection{Future Work}

Based on the results of this thesis, the following future works can be undertaken as:

- Combining proposed PMOR with High-order NILT:

High order NILT uses the same number of poles as the original NILT, but also uses the information of sensitivity of the system to generate the time response. High-order NILT will increase accuracy significantly while maintaining the same computational cost. Hence an adaptive PMOR preserving the moments of sensitivity should be developed.

- Extending the proposed PMOR to active circuit:

A stable PRIMA based model-order reduction to active circuit has been established in [38]. By integration the proposed PMOR algorithm with [38] can expand the horizon of variability analysis algorithms to include nonlinear elements. 


\section{List of References}

[1] F. Ferranti, M. S. Nakhla, G. Antonini, T. Dhaene, L. Knockaert, and A. E. Ruehli, "multipoint full-wave model order reduction for delayed PEEC models with large delays," vol. 53, pp. 959-967, Nov 2011.

[2] P. K. Gunupudi, R. Khazaka, M. S. Nakhla, T. Smy, and D. Celo, "Passive parameterized time-domain macromodels for high-speed transmission-line networks," IEEE Transactions on Microwave Theory and Techniques, vol. 51, no. 12, pp. 2347-2354, 2003.

[3] L. Daniel, O. C. Siong, L. S. Chay, K. H. Lee, and J. White, "A multiparameter moment-matching model-reduction approach for generating geometrically parameterized interconnect performance models," vol. 23, pp. 678-693, May 2004.

[4] P. Benner, S. Gugercin, and K. Willcox, "A survey of projection-based model reduction methods for parametric dynamical systems," SIAM Review, vol. 57, pp. 483-531, Nov. 2015.

[5] Y. Tao, M. Farhan, B. Nouri, and M. S. Nakhla, "Efficient variability analysis using parameterized model-order reduction," in Proc. IEEE MTT-S International Microwave Symposium (IMS-2016), (San Francisco, CA), May 2016.

[6] Y. Tao, B. Nouri, M. Nakhla, and R. Achar, "Efficient time-domain variability analysis using parameterized model-order reduction," in 2016 IEEE 20th Workshop on Signal and Power Integrity (SPI), pp. 1-4, May 2016.

[7] J. Vlach and K. Singhal, Computer methods for circuit analysis and design. Springer Science \& Business Media, 1983.

[8] E. Gad, M. Nakhla, R. Achar, and Y. Zhou, "-stable and-stable high-order integration methods for solving stiff differential equations," IEEE Transactions on Computer-Aided Design of Integrated Circuits and Systems, vol. 28, no. 9, pp. 1359-1372, 2009. 
[9] Y. Zhou, E. Gad, M. S. Nakhla, and R. Achar, "Structural characterization and efficient implementation techniques for-stable high-order integration methods," IEEE Transactions on Computer-Aided Design of Integrated Circuits and Systems, vol. 31, no. 1, pp. 101-108, 2012.

[10] M. Farhan, E. Gad, M. Nakhla, and R. Achar, "Efficient transient simulation of transmission lines and distributed circuits using high-order stable methods," in Microwave Symposium Digest (MTT), 2012 IEEE MTT-S International, pp. 13, IEEE, 2012.

[11] M. A. Farhan, E. Gad, M. S. Nakhla, and R. Achar, "New method for fast transient simulation of large linear circuits using high-order stable methods," IEEE Transactions on Components, Packaging and Manufacturing Technology, vol. 3, no. 4, pp. 661-669, 2013.

[12] G. G. Dahlquist, "A special stability problem for linear multistep methods," BIT Numerical Mathematics, vol. 3, no. 1, pp. 27-43, 1963.

[13] W. H. Schilders, H. A. Van der Vorst, and J. Rommes, Model order reduction: theory, research aspects and applications, vol. 13. Springer, 2008.

[14] A. C. Antoulas, Approximation of large-scale dynamical systems, vol. 6. Siam, 2005.

[15] M. Celik, L. Pileggi, and A. Odabasioglu, IC interconnect analysis. Springer Science \& Business Media, 2002.

[16] S. Tan and L. He, Advanced model order reduction techniques in VLSI design. Cambridge University Press, 2007.

[17] P. Benner, M. Hinze, and E. J. W. Ter Maten, Model reduction for circuit simulation. Springer, 2011.

[18] L. T. Pillage and R. A. Rohrer, "Asymptotic waveform evaluation for timing analysis," IEEE Transactions on Computer-Aided Design of Integrated Circuits and Systems, vol. 9, no. 4, pp. 352-366, 1990.

[19] E. Chiprout and M. S. Nakhla, "Asymptotic waveform evaluation," in Asymptotic Waveform Evaluation, pp. 15-39, Springer, 1994.

[20] E. J. Grimme, Krylov projection methods for model reduction. PhD thesis, Citeseer, 1997. 
[21] A. Odabasioglu, M. Celik, and L. T. Pileggi, "Prima: passive reduced-order interconnect macromodeling algorithm," in Proceedings of the 1997 IEEE/ACM international conference on Computer-aided design, pp. 58-65, IEEE Computer Society, 1997.

[22] P. Gunupudi and M. Nakhla, "Multi-dimensional model reduction of vlsi interconnects," in Custom Integrated Circuits Conference, 2000. CICC. Proceedings of the IEEE 2000, pp. 499-502, IEEE, 2000.

[23] J. W. Demmel, Applied numerical linear algebra. Siam, 1997.

[24] L. Daniel, O. C. Siong, L. S. Chay, K. H. Lee, and J. White, "A multiparameter moment-matching model-reduction approach for generating geometrically parameterized interconnect performance models," IEEE Transactions on Computer-Aided Design of Integrated Circuits and Systems, vol. 23, no. 5, pp. 678-693, 2004.

[25] P. Benner and L. Feng, "A robust algorithm for parametric model order reduction based on implicit moment matching," in Reduced order methods for modeling and computational reduction, vol. 9, ch. 6, pp. 159-185, New York: Springer, 2014.

[26] I. M. Elfadel and D. D. Ling, "A block rational arnoldi algorithm for multipoint passive model-order reduction of multiport rlc networks," in Proceedings of the 1997 IEEE/ACM international conference on Computer-aided design, pp. 66-71, IEEE Computer Society, 1997.

[27] E. O. Brigham and E. O. Brigham, The fast Fourier transform, vol. 7. PrenticeHall Englewood Cliffs, NJ, 1974.

[28] A. R. Djordjevic, K. S. Tapan, et al., "Analysis of time response of lossy multiconductor transmission line networks," in IEEE Trans. Microwave Theory Tech, Citeseer, 1987.

[29] J. R. Griffith and M. S. Nakhla, "Time-domain analysis of lossy coupled transmission lines," IEEE Transactions on microwave theory and techniques, vol. 38, no. 10 , pp. 1480-1487, 1990.

[30] R. Griffith and M. S. Nakhla, "Mixed frequency/time domain analysis of nonlinear circuits," IEEE Trans. Computer-Aided Design, vol. 11, no. 8, pp. 1032-1043, 1992. 
[31] T. K. Tang and M. S. Nakhla, "Analysis of high-speed vlsi interconnects using the asymptotic waveform evaluation technique," IEEE transactions on computeraided design of integrated circuits and systems, vol. 11, no. 3, pp. 341-352, 1992.

[32] E. Chiprout and M. S. Nakhla, "Analysis of interconnect networks using complex frequency hopping (cfh)," IEEE Transactions on Computer-Aided Design of Integrated Circuits and Systems, vol. 14, no. 2, pp. 186-200, 1995.

[33] J. Vlach and K. Singhal, Computer Methods for Circuit Analysis and Design. New York: Van Nostrand Reinhold, 1983.

[34] K. Singhal and J. Vlach, "Computation of time domain response by numerical inversion of the laplace transform," vol. 299, pp. 109-126, Feb. 1975.

[35] J. Griffith and M. Nakhla, "Time-domain analysis of lossy coupled transmission lines," vol. 38, pp. 1480-1487, Oct. 1990.

[36] R. Griffith, "Mixed frequency/time domain analysis of high-speed interconnects," m.a.sc. thesis, Carleton University, Ottawa, Canada, July 1993.

[37] K. Singhal, J. Vlach, and M. Nakhla, "Absolutely stable, high-order method for time domain solution of networks," AEU-INTERNATIONAL JOURNAL OF ELECTRONICS AND COMMUNICATIONS, vol. 30, no. 4, pp. 157-166, 1976.

[38] X. Deng, B. Nouri, and M. S. Nakhla, "Stability preserving algorithm for model order reduction of active networks," in Electrical Performance of Electronic Packaging and Systems (EPEPS), 2015 IEEE 24th, pp. 181-184, Oct 2015.

[39] R. Achar and M. S. Nakhla, "Simulation of high-speed interconnects," Proceedings of the IEEE, vol. 89, no. 5, pp. 693-728, 2001.

[40] M. Celik, L. Pileggi, and A. Odabasioglu, IC Interconnect Analysis. Norwell, MA: Kluwer, 2002. 


\section{Appendix A}

\section{Arnoldi Algorithm}

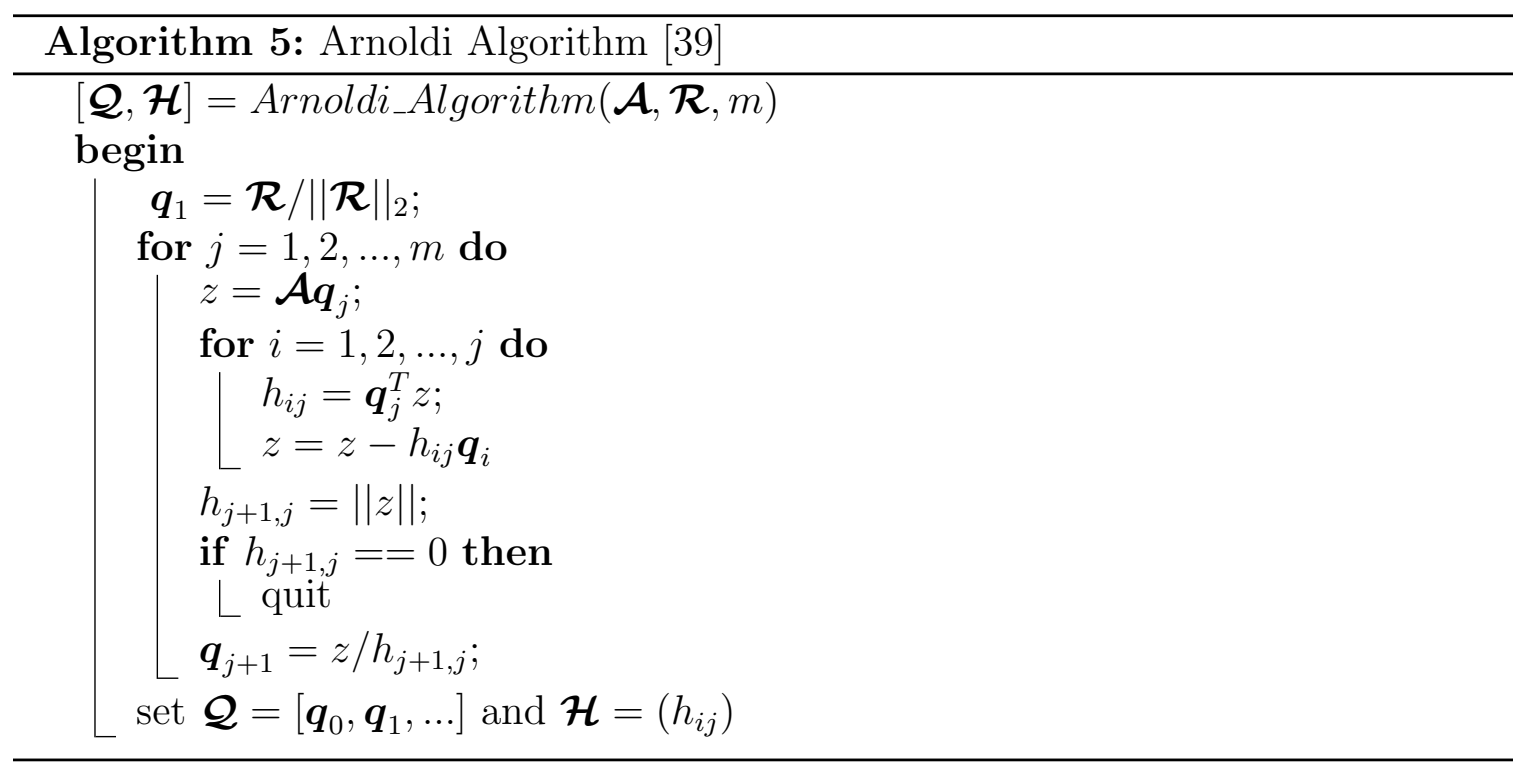




\section{Appendix B}

\section{Block Arnoldi Algorithm}

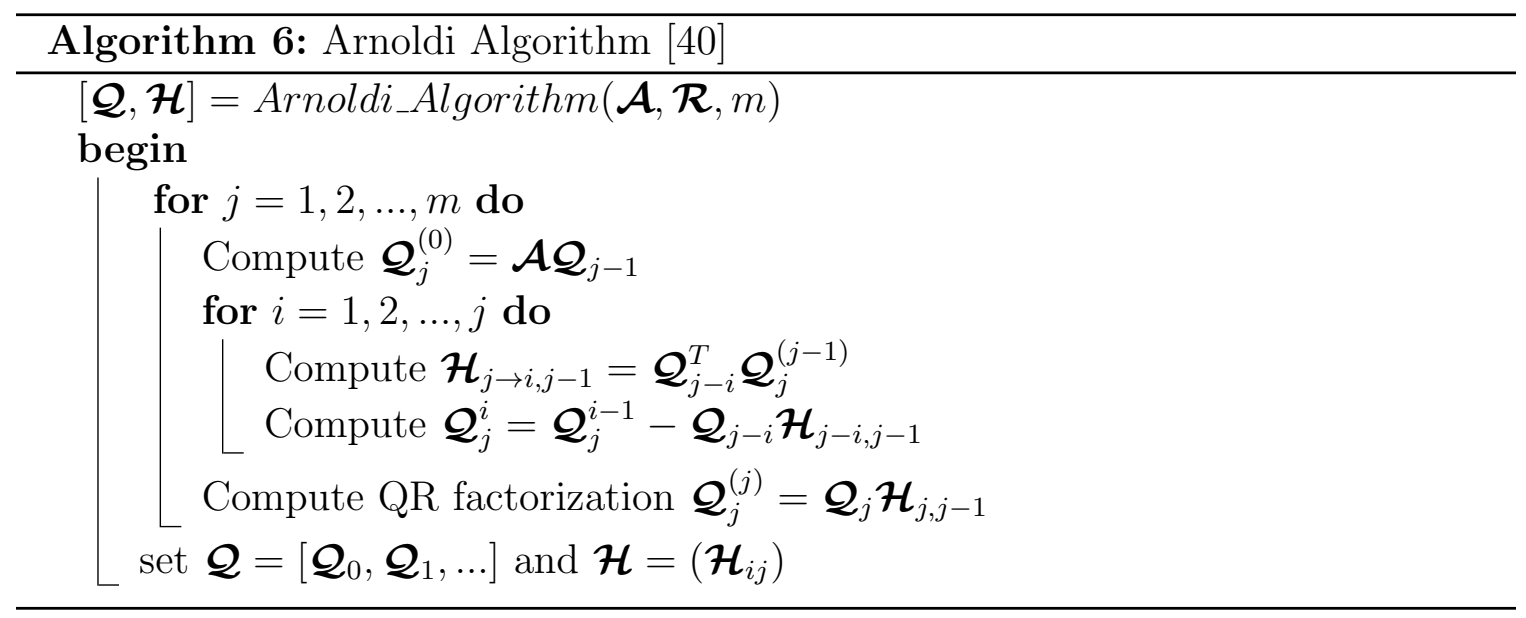




\section{Appendix $\mathrm{C}$}

\section{Orthogonal Matrix Calculation for Parametric System}

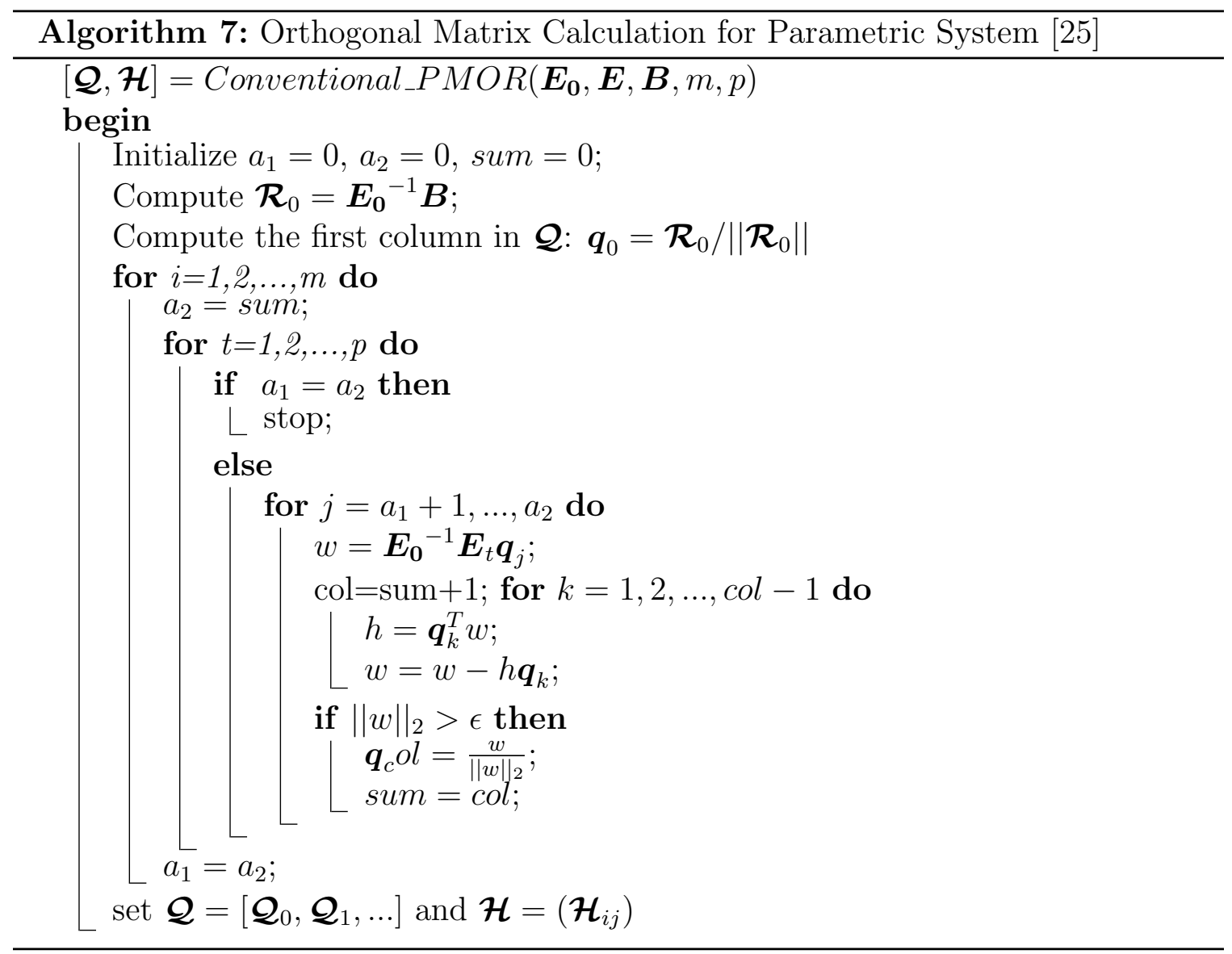




\section{Appendix D}

\section{Stability of NILT}

NILT can be as an A-stable integration method [37]. This chapter will have a brief overview of stability property of NILT.

The differential equation used as examining the stability property of an integration method is

$$
x^{\prime}=\lambda x
$$

where $\lambda$ is either real or complex constant with negative real part. The solution of this equation in Laplace domain is

$$
X=\frac{x_{0}}{s-\lambda}
$$

where $x_{0}$ is the initial condition. Substituting $\mathrm{X}$ into NILT with a step size $\mathrm{t}=\mathrm{h}$,

$$
x_{1}=-\frac{1}{h} \sum_{i=1}^{M} \frac{K_{i} x_{0}}{\frac{z_{i}}{h}-\lambda}=x_{0} \sum_{i=1}^{M} \frac{K_{i}}{h \lambda-z_{i}}
$$

Using $x_{1}$ as initial condition and calculate $x_{2}$,

$$
x_{2}=x_{1} \sum_{i=1}^{M} \frac{K_{i}}{h \lambda-z_{i}},
$$


Therefore the response at $\mathrm{n}$ steps will be

$$
x_{n}=x_{n-1} \sum_{i=1}^{M} \frac{K_{i}}{h \lambda-z_{i}}=x_{0}\left(\sum_{i=1}^{M} \frac{K_{i}}{h \lambda-z_{i}}\right)^{n} .
$$

NILT will be A-stable only if

$$
\lim _{n \rightarrow \infty} x_{n}=0 .
$$

To satisfy this criteria,

$$
\left(\sum_{i=1}^{M} \frac{K_{i}}{h \lambda-z_{i}}\right)^{n}=e^{n(h \lambda)} \leq 1 .
$$

Comparing equation D.7 with equation 5.3, as long as $M>N$ and step size $h>0$, this integration will be A-stable when real part of $\lambda$ is negative. 


\section{Appendix E}

\section{Error Estimation of NILT}

The truncation error of NILT, which has been proved in [37], is

$$
\Psi(N, M)=\frac{d_{N+M+1}\left(1-R_{N, M}(z=0)\right)}{(N+M+1) !} t^{N+M+1},
$$

where

$$
d_{N+M+1}=\left.v^{(N+M+1)}(t)\right|_{t=0} .
$$

The truncation error of NILT is caused by Padé approximation and it is function of $t^{N+M+1}$. The error goes high when $t \gg 0$. 